\title{
Lattice Boltzmann phase-field modeling of thermocapillary flows in a confined microchannel
}

\author{
Haihu Liu ${ }^{\mathrm{a}, *}$, Albert J. Valocchi ${ }^{\mathrm{a}}$, Yonghao Zhang ${ }^{\mathrm{b}}$, Qinjun Kang ${ }^{\mathrm{c}}$ \\ a Department of Civil \& Environmental Engineering, University of Illinois at Urbana-Champaign, Urbana, IL 61801, USA \\ b Department of Mechanical \&' Aerospace Engineering, University of Strathclyde, Glasgow G1 1XJ, UK \\ ${ }^{c}$ Earth and Environmental Sciences Division, Los Alamos National Laboratory, Los Alamos, NM 87545, USA
}

\section{A R T I C L E I N F O}

\section{Article history:}

Received 31 January 2013

Received in revised form 1 August 2013

Accepted 22 August 2013

Available online 12 September 2013

\section{Keywords:}

Phase-field model

Lattice Boltzmann method

Thermocapillary flow

Microfluidics

Surface wettability

Droplet dynamics

\begin{abstract}
A B S T R A C T
To understand how thermocapillary forces manipulate the droplet motion in a confined microchannel, a lattice Boltzmann phase-field model is developed to simulate immiscible thermocapillary flows with consideration of fluid-surface interactions. Based on our recent work of Liu et al., 2013 [54], an interfacial force of potential form is proposed to model the interfacial tension force and the Marangoni stress. As only the first-order derivatives are involved, the proposed interfacial force is easily combined with the wetting boundary condition to account for fluid-surface interactions. The hydrodynamic equations are solved using a multiple-relaxation-time algorithm with the interfacial force treated as a forcing term, while an additional convection-diffusion equation is solved by a passive-scalar approach to obtain the temperature field, which is coupled to the interfacial tension by an equation of state. The model is first validated against analytical solutions for the thermocapillary-driven convection in two superimposed fluids at negligibly small Reynolds and Marangoni numbers. It is then demonstrated to produce the correct equilibrium contact angle for a binary fluid with different viscosities when a constant interfacial tension is taken into account. Finally, we numerically simulate the thermocapillary flows for a microfluidic droplet adhering on a solid wall and subject to a simple shear flow when a laser is applied to locally heat the fluids, and investigate the influence of contact angle and fluid viscosity ratio on the droplet dynamical behavior. The droplet motion can be completely blocked provided that the contact angle exceeds a threshold value, below which the droplet motion successively undergoes four stages: constant velocity, deceleration, acceleration, and approximately constant velocity. When the droplet motion is completely blocked, three steady vortices are clearly visible, and the droplet is fully filled by two counter-rotating vortices with the smaller one close to the external vortex. The thermocapillary convection is strengthened with decreasing viscosity ratio of the droplet to the carrier fluid. For low viscosity ratios, the droplet motion is completely blocked and exhibits the similar behavior, but the structure of the internal vortices is more complicated at the lowest viscosity ratio. For high viscosity ratios, the droplet motion is partially blocked and undergoes a series of complex transitions, which can be explained as a result of the dynamically varying Marangoni forces.
\end{abstract}

(C) 2013 Elsevier Inc. All rights reserved.

\footnotetext{
* Corresponding author.

E-mail address: haihuliu@illinois.edu (H. Liu).
} 


\section{Introduction}

Microfluidic technology has attracted significant interest owing to its diverse applications in biotechnology, chemistry, pharmaceuticals and the life sciences. It has many advantages such as high throughput, short analysis time, small volume and high sensitivity. Recently, a multiphase fluidic approach, so-called droplet-based microfluidics, which creates discrete volumes with the use of immiscible phases has emerged as a promising flexible platform for microfluidic functions. It allows for independent control of each droplet, thus generating microreactors that can be individually transported, mixed, and analyzed. As samples/reagents are confined in the droplets, it can avoid sample dilution caused by Taylor dispersion, and eliminate surface adsorption and cross sample contamination [1]. In the past decade, microfluidic droplets have been widely applied to protein crystallization [2,3], drug delivery [4,5], material synthesis [6,7], as well as single-molecule/cell analysis $[8,9]$. It is essential to manipulate droplets in a precise and flexible manner in droplet-based microfluidic applications. In a microfluidic device, due to its intrinsically small dimensions, the effect of the interfacial tension, fluid viscosity, and surface wettability becomes dominant compared to the body forces such as buoyancy or inertia force. Therefore, the interfacial flow control is of great importance to manipulate the droplet behavior and optimize the performance of droplet-based microdevices.

The most commonly encountered droplet manipulations include droplet generation, fission, fusion, mixing and sorting [10]. Various approaches that cover a broad range of physical principles have been developed to achieve these manipulations, including electrowetting on dielectric (EWOD) [11,12], dielectrophoresis (DEP) [13,14], hydrodynamic stress [15-17], thermocapillary force [18,19], surface acoustic wave [20], magnetic force [21,22], and optical forces [23,24]. Among these approaches, optical forces provide a promising route towards microactuation because light fields can be easily focused to micrometric spots, and they are contactless and dynamically reconfigurable, without the need for any special microfabrication or moving parts [25]. However, optical forces are typically in the range of $p N$, which are often too weak to counteract hydrodynamic forces acting on moving droplets. In the recent years, an optical alternative based on the production of localized thermocapillary stresses (also known as Marangoni effect [26]) at the droplet interface has been demonstrated. In the presence of surfactant, the magnitude of thermocapillary forces induced by the localized heating from a laser can reach $\mu N$ range [27], so they are more effective than the optical forces themselves for droplet manipulation.

Thermocapillary convection is a phenomenon of fluid movement that arises as a consequence of the variation of interfacial tension at the interface caused by temperature differences. In most fluid systems, the interfacial tension is a decreasing function of the temperature; in surfactant stabilized systems, however, the interfacial tension may increase almost linearly with increasing temperature [27,28]. In any case, the induced thermocapillary stresses lead to the movement of droplets from the regions of high interfacial tension to the regions of low interfacial tension. The thermocapillary motion of droplets plays an important role in many natural physical processes as well as numerous industrial activities, particularly in space material processing and many other engineering and scientific applications under microgravity conditions where sedimentation and gravity-driven convection are largely eliminated. Thus, it attracts an increasing amount of research interest worldwide along with the progress of human space technology. For a comprehensive review of this topic, readers can refer to the excellent book by Subramanian and Balasubramanian [29]. Since thermocapillary convection has an interfacial origin, it is particularly suitable to drive flows at small scales, as in microchannels. To date, optically-induced thermocapillary forces have been used for demonstrating many droplet-based microfluidic phenomena. It was found that a non-wetting droplet resting on a substrate can move under laser-driven thermocapillary stresses [30]. Baroud et al. [27] showed that a water droplet, transported by an immiscible oil flow in a cross-shaped microchannel, may be blocked if submitted to a focused laser spot. This blocking may last several seconds (typically $\sim 2 \mathrm{~s}$ ), indicating that the drag force from the viscous oil is balanced (at least temporarily) by the thermocapillary force generated by the laser heating. They also further showed that the strength of the blocking force increases with reduced droplet size and may become several orders of magnitude larger than forces generated by dielectrophoresis. The optically-induced blocking force was also combined with the geometry of the microchannel to realize various droplet manipulations including mixing, sorting, fission, fusion, sampling and switching $[25,31]$.

Although experimental studies have helped to understand thermocapillary flows in microfluidic devices, it is still very difficult to carry out precise experimental measurements of the local temperature and flow fields during the transport process of a droplet. Thus, the physical mechanism and dynamic behavior of droplet migration are still unclear and remain open questions. Numerical modelling and simulations can be very instrumental in enhancing our understanding of thermocapillary flows at microscale, complementary to experimental studies. However, numerical simulation of thermocapillary flows is a challenging task, where the capillary effect usually plays a dominant role. Discretization errors in calculation of interfacial forces may generate unphysical spurious velocities which can cripple the velocity field in the whole computational domain. Minimizing the spurious velocities at the interface still remains a major challenge for numerical models and algorithms. Additionally, contact-line dynamics plays an important role when the droplet touches a solid wall in microfluidic channels. Due to its inherent multiscale nature, contact-line dynamics is still a long-standing physical problem to be resolved [32], thereby posing an additional numerical challenge. Finally, due to the strong dependence of interfacial tension on temperature, the temperature fluctuations result in non-uniform interfacial tension forces and Marangoni stresses that affect the flowfield at the interface, which in turn alter the interfacial temperature distribution through the induced interfacial flows. While the front-tracking method is not suitable for simulating droplet breakup and coalescence because the interface must be manually ruptured based upon some ad-hoc criteria [33], interface capturing methods such as volume-of-fluid and level 
set methods will suffer from numerical instability at the interface region when the interfacial tension becomes a dominant factor in microdroplet behavior [34]. Microscopically, the interface between different phases and the contact-line dynamics on the solid surface are due to interparticle forces/interactions [35]. Thus, mesoscopic level models are expected to describe accurately the thermocapillary flows in a confined microchannel.

The lattice Boltzmann method (LBM), as a mesoscopic numerical method, has been developed into a promising alternative to traditional computational fluid dynamics (CFD) for simulating complex fluid flows in recent years. The LBM has several advantages over the traditional CFD methods such as the ability to be programmed on parallel computers and the ease in dealing with complex boundaries [36]. Besides, its kinetic nature provides many of the advantages of molecular dynamics, making the LBM particularly useful in simulating complex interfacial dynamics. Historically, the first multiphase LBM model was proposed by Gunstensen et al. [37] based on a lattice gas automation (LGA) model [38]. Since then, many multiphase LBM models have been developed. These models can be classified into four types, i.e., the colorfluid model [37,39-41], the interparticle-potential model [42-44], the phase-field-based model [45-49], and the mean-field theory model [50]. For a comprehensive review of these models, interested readers may refer to Refs. [36,51,52]. These multiphase LBM models mainly focus on interfacial flows with a constant interfacial tension, so they are incapable of simulating thermocapillary flows around moving and stationary interfaces except our recently improved color-fluid model [53] and phase-field-based lattice Boltzmann finite-difference model [54]. In our improved color-fluid model, a concept of continuum surface force (CSF) is used to model the interfacial tension force and Marangoni stress, and the phase segregation is achieved through the recoloring algorithm proposed by Latva-Kokko and Rothman [55]. In the phase-field-based lattice Boltzmann finite-difference model, an interfacial force of stress form is analytically derived using the free energy theory, and the hydrodynamic equations, including the Navier-Stokes equations (NSEs) and the Cahn-Hilliard equation (CHE), are solved through an improved lattice Boltzmann equation (LBE) method originally proposed by Lee and Liu [49] while the temperature equation is solved through a combination of the finite-difference method and the Runge-Kutta method. However, the both models can only simulate thermocapillary flows with droplets suspended in a carrier fluid, away from the wall boundary. In addition, the calculation of interfacial force needs to estimate not only the gradient of phase-field but also its derivatives, which usually introduces incompatible discretization errors [56-58], leading to large spurious currents in the vicinity of the wall boundary when the surface wettability is enforced. These deficiencies largely limit their prospect for droplet-based microfluidic applications.

In this paper, a lattice Boltzmann phase-field model is developed to simulate immiscible thermocapillary flows in a confined microchannel. Using a phase-field methodology, an interfacial force of potential form is derived analytically to model the interfacial tension force and the Marangoni stress. The proposed interfacial force is also combined with a cubic wetting boundary condition to account for fluid-surface interactions, i.e., the contact-line dynamics. The fluid flows are solved through a multiple-relaxation-time (MRT) LBM with the interfacial force treated as a forcing term $[59,60]$ while the phase interface is captured following a strategy proposed by Pooley et al. [32], which can enhance the numerical stability for solving a binary fluid with large viscosity difference and produce correct equilibrium contact angles. The temperature field, which is coupled to the interfacial tension by the equation of state, is also solved in the framework of LBM, where thermal boundary conditions are imposed by a general halfway bounce-back scheme recently proposed by Zhang et al. [61]. The capability and accuracy of this model are first tested by two benchmark cases with analytical solutions. It is then used to simulate the droplet dynamical behavior for a semi-circular droplet adhering on the bottom wall in a microchannel and subject to a simple shear flow when a laser heating is applied. We show how the laser light blocks the droplet motion and investigate the influence of contact angle and fluid viscosity ratio on the droplet motion. This study can provide an in-depth understanding of thermocapillary flows for droplets in contact with solid walls in confined microfluidic devices and facilitate design to precisely control droplet behavior.

\section{Methodology}

\subsection{Phase-field theory and governing equations for hydrodynamics}

We consider here an incompressible system of two nominally immiscible Newtonian fluids in contact with each other and with a solid surface. The order parameter $\phi$ is introduced to identify the regions where two fluid flows occur: $\phi=-1$ is occupied by fluid ' $\mathrm{A}$ ' and $\phi=1$ by fluid ' $\mathrm{B}$ '. The interface is represented by $\phi=0$ with an interfacial layer of thickness $\epsilon$. Denoting the fluid domain by $\Omega$ and the solid surface by $\partial \Omega$, one can write the total free energy of the system as [62]

$$
\mathcal{F}(\phi)=\int_{\Omega}\left(\Psi(\phi)+\frac{\epsilon^{2}}{2}|\nabla \phi|^{2}\right) d \Omega+\int_{\partial \Omega} f_{w}(\phi) d S,
$$

where $\Psi(\phi)$ is the bulk free energy density and takes a double-well form $\Psi(\phi)=\frac{1}{4}\left(\phi^{2}-1\right)^{2}$. The term $\frac{\epsilon^{2}}{2}|\nabla \phi|^{2}$ accounts for the excess free energy in the interfacial region, where $\epsilon$ is the interface thickness. The second integral represents the wall free energy due to the fluid-surface interactions, where $f_{w}$ is determined by the order parameter at the solid surface and the dimensionless wetting potential $\Omega_{c}$ [62],

$$
f_{w}(\phi)=\frac{\epsilon\left(3 \phi-\phi^{3}\right)}{3 \sqrt{2}} \Omega_{c}
$$


where $\Omega_{c}$ is related to the equilibrium contact angle $\theta^{e q}$ by Young's equation:

$$
\cos \left(\theta^{e q}\right)=\frac{\sigma_{B w}-\sigma_{A w}}{\sigma}=-\Omega_{c},
$$

where $\sigma$ is the interfacial tension between fluid $\mathrm{A}$ and $\mathrm{B}$, and $\sigma_{A w}$ (or $\sigma_{B w}$ ) is the interfacial tension between the fluid phase $\mathrm{A}$ (or $\mathrm{B}$ ) and the solid surface.

A variational procedure is applied to the free energy functional, i.e. Eq. (1), and leads to

$$
\begin{aligned}
\delta \mathcal{F} & =\left.\frac{d}{d r} \mathcal{F}(\phi+r \delta \phi)\right|_{r=0}=\int_{\Omega}\left[\delta \phi \Psi^{\prime}(\phi)+\epsilon^{2} \nabla \phi \cdot \nabla \delta \phi\right] d \Omega+\int_{\partial \Omega} f_{w}^{\prime}(\phi) \delta \phi d S \\
& =\int_{\Omega}\left[\Psi^{\prime}(\phi)-\epsilon^{2} \nabla^{2} \phi\right] \delta \phi d \Omega+\int_{\partial \Omega}\left[\epsilon^{2}\left(\mathbf{n}_{w} \cdot \nabla \phi\right)+f_{w}^{\prime}(\phi)\right] \delta \phi d S \\
& =\int_{\Omega}\left[\Psi^{\prime}(\phi)-\epsilon^{2} \nabla^{2} \phi\right] \delta \phi d \Omega,
\end{aligned}
$$

where a natural boundary condition (also called cubic boundary condition in Ref. [49]) is imposed on the solid substrate $\partial \Omega$ :

$$
\mathbf{n}_{w} \cdot \nabla \phi=-\frac{1}{\epsilon^{2}} f_{w}^{\prime}(\phi)=\frac{\phi^{2}-1}{\sqrt{2} \epsilon} \Omega_{c} .
$$

Here $\mathbf{n}_{w}$ is the outward-pointing unit vector normal to the solid surface. This cubic boundary condition has been widely used to simulate two-phase flows with moving contact lines [62-64,49]. It was demonstrated numerically that such a boundary condition can eliminate the spurious variation of order parameter at solid boundaries, thereby allowing us to better capture correct physics than its lower-order counterparts [64].

From Eq. (4) we can get the chemical potential $\mu$, which is defined as the variational derivative of the free energy with respect to the order parameter,

$$
\mu=\frac{\delta \mathcal{F}}{\delta \phi}=\Psi^{\prime}(\phi)-\epsilon^{2} \nabla^{2} \phi=\phi^{3}-\phi-\epsilon^{2} \nabla^{2} \phi .
$$

The equilibrium interface profile can be obtained from Eq. (6) at $\mu=0$. The one-dimensional solution of this equation is

$$
\phi(z)=\tanh \left(\frac{z}{\sqrt{2} \epsilon}\right)
$$

where $z$ is the spatial location normal to the interface $(z=0)$.

The time evolution of the diffuse interface is described by the convective CHE, in which the local diffusion flux is proportional to the gradient of the chemical potential:

$$
\frac{\partial \phi}{\partial t}+\mathbf{u} \cdot \nabla \phi=\nabla \cdot\left(M_{\phi} \nabla \mu\right)
$$

where $\mathbf{u}$ is the flow velocity, and $M_{\phi}>0$ is the mobility.

It is well known that the expression for the stress jump across the interface $\Sigma$ is given by

$$
[\mathbf{T} \cdot \mathbf{n}]_{\Sigma}=\left.\mathbf{T} \cdot \mathbf{n}\right|_{\Sigma, B}-\left.\mathbf{T} \cdot \mathbf{n}\right|_{\Sigma, A}=\sigma \kappa \mathbf{n}-\nabla_{S} \sigma,
$$

where $\mathbf{T}=-p \mathbf{I}+\eta\left(\nabla \mathbf{u}+\nabla \mathbf{u}^{T}\right)$ is the stress tensor, $p$ is the pressure, $\eta$ is the dynamic viscosity, $\mathbf{I}$ is the second-order identity tensor, $\mathbf{n}$ is the unit vector normal to $\Sigma$ and directing towards fluid $\mathrm{B}, \kappa=\nabla \cdot \mathbf{n}$ is the local interface curvature and $\nabla_{S}=(\mathbf{I}-\mathbf{n} \otimes \mathbf{n}) \cdot \nabla$ is the surface gradient operator. The first term on the right-hand side of Eq. (9) is the interfacial tension force and the second term is the Marangoni stress. In order to induce the local stress jump across the interface, a volume-distributed interfacial force $\mathbf{F}_{S}$, should be added in the momentum equation as an additional body force. The interfacial force is

$$
\mathbf{F}_{S}=\left(-\sigma \kappa \mathbf{n}+\nabla_{S} \sigma\right) \delta_{\Sigma},
$$

where $\delta_{\Sigma}$ is the Dirac delta function used to localize the force explicitly at the interface, which should satisfy

$$
\int_{-\infty}^{\infty} \delta_{\Sigma} d z=1
$$

in order to recover properly the stress jump condition, Eq. (9), in the sharp-interface limit. 
By defining $\mathbf{n}=\frac{\nabla \phi}{|\nabla \phi|}$ and $\delta_{\Sigma}=\frac{3 \sqrt{2}}{4} \epsilon|\nabla \phi|^{2}$, an interfacial force of stress form was derived from Eq. (10) and can be written as [54]

$$
\mathbf{F}_{S}=\frac{3 \sqrt{2}}{4} \epsilon \nabla \cdot\left[\sigma(T)\left(|\nabla \phi|^{2} \mathbf{I}-\nabla \phi \otimes \nabla \phi\right)\right]
$$

However, one needs to approximate not only the gradient of $\phi$ but also the derivatives of the gradient in order to calculate the above interfacial force. This may induce undesirable discretization errors at the solid surface when the wetting boundary condition, i.e. Eq. (5), is enforced. It is therefore not surprising that none of the existing studies uses the interfacial force of stress form to simulate the contact-line dynamics in the phase-field approaches [65,66,63,67,49,64]. Note that these studies only consider a constant interfacial tension, so they cannot simulate thermocapillary flows around moving and stationary droplets, which are crucial for many microgravity applications and opto-thermal manipulation of droplets in microfluidic devices.

Using Eq. (6) and the following equalities [68,54]:

$$
\begin{aligned}
& |\nabla \phi|^{2}=\frac{1}{2 \epsilon^{2}}\left(\phi^{2}-1\right)^{2}, \\
& \nabla \cdot(\nabla \phi \otimes \nabla \phi)=\frac{1}{2} \nabla\left(|\nabla \phi|^{2}\right)+\nabla^{2} \phi \nabla \phi,
\end{aligned}
$$

we can transform the interfacial force equation (12) into a different but equivalent form:

$$
\mathbf{F}_{S}=\frac{3 \sqrt{2}}{4} \epsilon\left[|\nabla \phi|^{2} \nabla \sigma-(\nabla \sigma \cdot \nabla \phi) \nabla \phi+\frac{\sigma}{\epsilon^{2}} \mu \nabla \phi\right],
$$

which is referred to the interfacial force of "potential form". Obviously, the discretization of gradient terms is only required in Eq. (15). This can relieve the numerical difficulty in estimating the derivatives of the gradient at the solid surface. In particular, if the interfacial tension is a constant, the interfacial force term can reduce to

$$
\mathbf{F}_{S}=\frac{3 \sqrt{2}}{4 \epsilon} \sigma \mu \nabla \phi,
$$

which is commonly used in literature. It is widely recognized that spurious velocities at the interfaces originate from incompatible discretizations of the interfacial force. The interfacial force of potential form is able to produce smaller discretization errors than its stress form [56-58], which is key to small spurious velocities.

In a thermocapillary flow, an equation of state is required to relate the interfacial tension to the temperature, which may be linear or nonlinear. For the sake of simplicity, we only consider a linear relation between the interfacial tension and the temperature $T$ in this study [69,53], i.e.,

$$
\sigma(T)=\sigma_{r e f}+\sigma_{T}\left(T-T_{r e f}\right),
$$

where $T_{r e f}$ is the reference temperature, $\sigma_{r e f}$ is the interfacial tension at $T_{r e f}, \sigma_{T}$ is the rate of change of interfacial tension with temperature, defined as $\sigma_{T}=\partial \sigma / \partial T$.

Substituting Eq. (17) into Eq. (15), we obtain the interfacial force as

$$
\mathbf{F}_{S}=\frac{3 \sqrt{2}}{4} \epsilon\left[\sigma_{T}|\nabla \phi|^{2} \nabla T-\sigma_{T}(\nabla T \cdot \nabla \phi) \nabla \phi+\frac{\sigma}{\epsilon^{2}} \mu \nabla \phi\right] .
$$

With the interfacial force $\mathbf{F}_{S}$ given by Eq. (18), the macroscopic governing equations for the incompressible fluid flows can be written as $[65,63,66,67]$

$$
\begin{aligned}
& \nabla \cdot \mathbf{u}=0, \\
& \rho\left(\frac{\partial \mathbf{u}}{\partial t}+\mathbf{u} \cdot \nabla \mathbf{u}\right)=-\nabla p+\nabla \cdot\left[\eta\left(\nabla \mathbf{u}+\nabla \mathbf{u}^{T}\right)\right]+\mathbf{F}_{S},
\end{aligned}
$$

where $\rho$ is the density of fluid mixture.

\subsection{Lattice Boltzmann method for hydrodynamic equations}

The NSEs, Eqs. (19) and (20), are solved using the MRT-LBM, while the CHE, Eq. (8), is solved through the standard singlerelaxation-time (SRT) LBM as suggested by Pooley et al. [32]. MRT-LBM has a number of advantages over the SRT-LBM in simulating various flow problems, including enhanced numerical instability, the ability to model thermohydrodynamics with adjustable Prandtl numbers [70], removing the viscosity-dependent velocity field [71], and minimization of the spurious velocities and reproducing the correct contact angle for simulating multiphase flows [32]. In this model, we need to define two particle distribution functions (PDFs) $f_{i}(\mathbf{x}, t)$ and $g_{i}(\mathbf{x}, t)$ on each lattice point, where $i$ is the lattice direction. The 
first distribution function is related to macroscopic fluid density $\rho$ and momentum $\mathbf{j}$, and the second distribution function captures the order parameter $\phi$

$$
\rho(\mathbf{x}, t)=\sum_{i} f_{i}(\mathbf{x}, t), \quad \mathbf{j}(\mathbf{x}, t)=\sum_{i} f_{i}(\mathbf{x}, t) \mathbf{e}_{i}+\frac{1}{2} \mathbf{F}_{S} \delta_{t}, \quad \phi(\mathbf{x}, t)=\sum_{i} g_{i}(\mathbf{x}, t),
$$

where $\mathbf{j}=\rho_{0} \mathbf{u}, \rho_{0}$ is the mean fluid density which is used to reduce compressibility effects in the model [72,71], and the fluid velocity $\mathbf{u}$ is defined effectively to carry some of the influence of the variable interfacial force; $\mathbf{e}_{i}$ is the lattice velocity vector defined as $\mathbf{e}_{0}=(0,0), \mathbf{e}_{1,3}=( \pm c, 0), \mathbf{e}_{2,4}=(0, \pm c), \mathbf{e}_{5,7}=( \pm c, \pm c)$, and $\mathbf{e}_{6,8}=(\mp c, \pm c)$ for a two-dimensional 9 -velocity model (D2Q9). The lattice speed $c$ is defined by $c=\delta_{x} / \delta_{t}$, where $\delta_{x}$ and $\delta_{t}$ are the lattice spacing and time step, respectively. The speed of sound $c_{s}$ can be related to $c$ by $c_{s}=c / \sqrt{3}$.

The evolution equations of the PDFs $f_{i}(\mathbf{x}, t)$ and $g_{i}(\mathbf{x}, t)$ are governed by

$$
\begin{aligned}
& f_{i}\left(\mathbf{x}+\mathbf{e}_{i} \delta_{t}, t+\delta_{t}\right)-f_{i}(\mathbf{x}, t)=\Omega_{i}^{f}+\delta_{t} \bar{F}_{i}, \\
& g_{i}\left(\mathbf{x}+\mathbf{e}_{i} \delta_{t}, t+\delta_{t}\right)-g_{i}(\mathbf{x}, t)=\Omega_{i}^{g},
\end{aligned}
$$

where $\Omega_{i}^{f}$ and $\Omega_{i}^{g}$ are the MRT collision operator for $f_{i}$ and the standard BGK collision operator for $g_{i}$, and are given by

$$
\begin{aligned}
& \Omega_{i}^{f}=-\left(\mathbf{M}^{-1} \mathbf{S M}\right)_{i j}\left[f_{j}(\mathbf{x}, t)-f_{j}^{e q}(\mathbf{x}, t)\right], \\
& \Omega_{i}^{g}=-\frac{1}{\tau_{g}}\left[g_{i}(\mathbf{x}, t)-g_{i}^{e q}(\mathbf{x}, t)\right],
\end{aligned}
$$

where $f_{i}^{e q}$ and $g_{i}^{e q}$ are the equilibrium distribution functions of $f_{i}$ and $g_{i}, \tau_{g}$ is the single relaxation parameter for $g_{i}$, $\mathbf{M}$ is a transformation matrix, and $\mathbf{S}$ is a diagonal relaxation matrix.

$\bar{F}_{i}$ in Eq. (22) represents the discrete forcing term accounting for the interfacial force acting on the fluid mixture, which is given by [59]

$$
\overline{\mathbf{F}}=\mathbf{M}^{-1}\left(\tilde{\mathbf{I}}-\frac{1}{2} \mathbf{S}\right) \mathbf{M} \tilde{\mathbf{F}}
$$

where $\tilde{\mathbf{I}}$ is a $9 \times 9$ unit matrix, $\overline{\mathbf{F}}=\left[\overline{F_{0}}, \overline{F_{1}}, \overline{F_{2}}, \overline{F_{3}}, \overline{F_{4}}, \overline{F_{5}}, \overline{F_{6}}, \overline{F_{7}}, \overline{F_{8}}\right]^{T}$, and $\tilde{\mathbf{F}}=\left[\tilde{F_{0}}, \tilde{F_{1}}, \tilde{F_{2}}, \tilde{F_{3}}, \tilde{F_{4}}, \tilde{F_{5}}, \tilde{F_{6}}, \tilde{F_{7}}, \tilde{F_{8}}\right]^{T}$.

The governing physics of LBM is determined through the hydrodynamic moments of the equilibrium distribution functions and the forcing terms. The moments of $f_{i}^{e q}, g_{i}^{e q}$ and $\tilde{F}_{i}$ are:

$$
\begin{aligned}
& \sum_{i} f_{i}^{e q}=\rho, \quad \sum_{i} f_{i}^{e q} e_{i \alpha}=\rho_{0} u_{\alpha}, \quad \sum_{i} f_{i}^{e q} e_{i \alpha} e_{i \beta}=\rho_{0} u_{\alpha} u_{\beta}+\rho c_{s}^{2} \delta_{\alpha \beta}, \\
& \sum_{i} f_{i}^{e q} e_{i \alpha} e_{i \beta} e_{i \gamma}=\rho_{0} c_{s}^{2}\left(\delta_{\alpha \beta} u_{\gamma}+\delta_{\alpha \gamma} u_{\beta}+\delta_{\beta \gamma} u_{\alpha}\right), \\
& \sum_{i} g_{i}^{e q}=\phi, \quad \sum_{i} g_{i}^{e q} e_{i \alpha}=\phi u_{\alpha}, \quad \sum_{i} g_{i}^{e q} e_{i \alpha} e_{i \beta}=\phi u_{\alpha} u_{\beta}+\Gamma \mu \delta_{\alpha \beta}, \\
& \sum_{i} \tilde{F}_{i}=0, \quad \sum_{i} \tilde{F}_{i} e_{i \alpha}=F_{S \alpha}, \quad \sum_{i} \tilde{F}_{i} e_{i \alpha} e_{i \beta}=u_{\alpha} F_{S \beta}+u_{\beta} F_{S \alpha} .
\end{aligned}
$$

A suitable choice for $f_{i}^{e q}, g_{i}^{e q}$ and $\tilde{F}_{i}$ that satisfies the constraints (27)-(29) is a power series expansion in terms of the velocity

$$
\begin{aligned}
& f_{i}^{e q}=w_{i}\left[\rho+\rho_{0}\left(\frac{\mathbf{e}_{i} \cdot \mathbf{u}}{c_{s}^{2}}+\frac{\mathbf{u u}:\left(\mathbf{e}_{i} \mathbf{e}_{i}-c_{s}^{2} \mathbf{I}\right)}{2 c_{s}^{4}}\right)\right], \\
& g_{i}^{e q}=w_{i}\left[G_{i}+\phi\left(\frac{\mathbf{e}_{i} \cdot \mathbf{u}}{c_{s}^{2}}+\frac{\mathbf{u} \mathbf{u}:\left(\mathbf{e}_{i} \mathbf{e}_{i}-c_{s}^{2} \mathbf{I}\right)}{2 c_{s}^{4}}\right)\right], \\
& \tilde{F}_{i}=w_{i}\left[\frac{\mathbf{e}_{i} \cdot \mathbf{F}_{S}}{c_{s}^{2}}+\frac{\mathbf{u} \mathbf{F}_{S}:\left(\mathbf{e}_{i} \mathbf{e}_{i}-c_{s}^{2} \mathbf{I}\right)}{2 c_{s}^{4}}\right],
\end{aligned}
$$

where the coefficient $G_{i}$ is given by

$$
G_{i}= \begin{cases}\Gamma \mu / c_{s}^{2} & (i>0) \\ {\left[\phi-\left(1-w_{0}\right) \Gamma \mu / c_{s}^{2}\right] / w_{0}} & (i=0),\end{cases}
$$

and $w_{i}$ is the weight factor with $w_{0}=4 / 9, w_{1-4}=1 / 9$ and $w_{5-8}=1 / 36$. 
The transformation matrix $\mathbf{M}$ is given explicitly as [73]

$$
\mathbf{M}=\left(\begin{array}{ccccccccc}
1 & 1 & 1 & 1 & 1 & 1 & 1 & 1 & 1 \\
-4 & -1 & -1 & -1 & -1 & 2 & 2 & 2 & 2 \\
4 & -2 & -2 & -2 & -2 & 1 & 1 & 1 & 1 \\
0 & 1 & 0 & -1 & 0 & 1 & -1 & -1 & 1 \\
0 & -2 & 0 & 2 & 0 & 1 & -1 & -1 & 1 \\
0 & 0 & 1 & 0 & -1 & 1 & 1 & -1 & -1 \\
0 & 0 & -2 & 0 & 2 & 1 & 1 & -1 & -1 \\
0 & 1 & -1 & 1 & -1 & 0 & 0 & 0 & 0 \\
0 & 0 & 0 & 0 & 0 & 1 & -1 & 1 & -1
\end{array}\right)
$$

which is designed to contain more physically relevant quantities, e.g. density, momentum, energy, and their fluxes. The diagonal relaxation matrix $\mathbf{S}$ is defined as

$$
\mathbf{S}=\operatorname{diag}\left[s_{0}, s_{1}, s_{2}, s_{3}, s_{4}, s_{5}, s_{6}, s_{7}, s_{8}\right],
$$

where the elements $s_{i}$ are the relaxation rates associated with each $f_{i}$. The parameters $s_{0}, s_{3}$ and $s_{5}$ correspond to the conserved moments (i.e., density and momentum) and are chosen as $s_{0}=s_{3}=s_{5}=1$. $s_{7}$ and $s_{8}$ are related to the kinematic viscosity $v$ by

$$
s_{7}=s_{8}=\frac{1}{\tau_{f}}, \quad \text { and } \quad v=\left(\tau_{f}-\frac{1}{2}\right) c_{s}^{2} \delta_{t} .
$$

Besides, symmetry requires that $s_{4}=s_{6}$. As a result, three independent parameters $s_{1}, s_{2}$ and $s_{4}\left(=s_{6}\right)$ can be freely adjusted to enhance the stability of MRT model [72,74,59,75]. Following the guidelines and suggestions in [73], we choose these free parameters as $s_{1}=1.63, s_{2}=1.14$ and $s_{4}=s_{6}=1.92$ in this study. It is worth noting that the MRT model will reduce to the BGK model if all $s_{i}$ in Eq. (35) are set to $1 / \tau_{f}$.

Employing the Chapman-Enskog multiscale analysis, Eqs. (22) and (23) can lead to the hydrodynamic equations (19), (20) and (8) with the pressure $p=\rho c_{s}^{2}$ in the limit of the low Mach number. The relaxation parameter $\tau_{g}$ is related to the mobility $M_{\phi}$ through [46,32]

$$
M_{\phi}=\delta_{t} \Gamma\left(\tau_{g}-\frac{1}{2}\right),
$$

where $\Gamma$ is a constant that appears in the equilibrium distribution function $g_{i}^{e q}$. As pointed out by Pooley et al. [32], it is not necessary to adopt an MRT approach for $g_{i}$ since one can simply set $\tau_{g}=1$ and independently use $\Gamma$ to control the mobility. To account for unequal viscosities of the two fluids, we define the viscosity $v$ as a linear function of the order parameter

$$
v(\phi)=\frac{1-\phi}{2} v_{A}+\frac{1+\phi}{2} v_{B}
$$

where $v_{A}$ and $v_{B}$ are the viscosities of the fluid $A$ and $B$, respectively.

\subsection{Lattice Boltzmann method for temperature equation}

If the viscous heat dissipation and compression work done by the pressure are negligible, the governing equation describing temperature field can be written as [53]

$$
\frac{\partial T}{\partial t}+\mathbf{u} \cdot \nabla T=\nabla \cdot(k \nabla T)+q_{T},
$$

where $k$ is the thermal conductivity, and $q_{T}$ is the heat source density. Following the definition of fluid viscosity given by Eq. (38), we define the thermal conductivity of mixture as

$$
k(\phi)=\frac{1-\phi}{2} k_{A}+\frac{1+\phi}{2} k_{B},
$$

where $k_{A}\left(k_{B}\right)$ is the thermal conductivity of fluid $\mathrm{A}(\mathrm{B})$. Instead of directly solving Eq. (39), the temperature equation is also modelled by a lattice Boltzmann equation for an independent temperature distribution function, which is known as the passive-scalar (PS) approach. Compared to its counterpart-the multi-speed (MS) approach, PS approach can overcome some inherent limitations, e.g., the numerical instability, the narrow range of temperature variation, and the fixed value of the Prandtl number [76]. The reliability of the PS approach for thermal flows has been verified by many benchmark studies [77, 76,78]. 


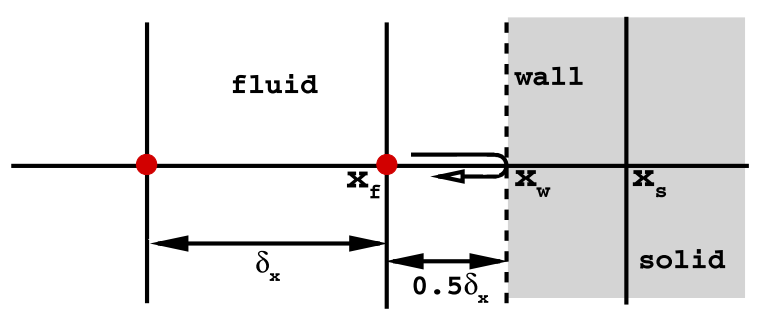

Fig. 1. A schematic to illustrate the location of the boundary nodes.

In order to simulate the evolution of temperature field, another distribution function $h_{i}$ is used:

$$
h_{i}\left(\mathbf{x}+\mathbf{e}_{i} \delta_{t}, t+\delta_{t}\right)-h_{i}(\mathbf{x}, t)=-\frac{1}{\tau_{h}}\left[h_{i}(\mathbf{x}, t)-h_{i}^{e q}(\mathbf{x}, t)\right]+\delta_{t} w_{i} q_{T},
$$

where $\tau_{h}$ is the dimensionless relaxation time related to the thermal conductivity as $k=\left(\tau_{h}-1 / 2\right) c_{s}^{2} \delta_{t} ; h_{i}^{e q}(\mathbf{x}, t)$ is the equilibrium distribution function in the $i$-th direction at the location $\mathbf{x}$ and the time $t$, which is chosen to recover the macroscopic temperature equation, i.e. Eq. (39),

$$
h_{i}^{e q}=w_{i} T\left[1+\frac{\mathbf{e}_{i} \cdot \mathbf{u}}{c_{s}^{2}}+\frac{\mathbf{u u}:\left(\mathbf{e}_{i} \mathbf{e}_{i}-c_{s}^{2} \mathbf{I}\right)}{2 c_{s}^{4}}\right],
$$

where the temperature $T$ is calculated by

$$
T(\mathbf{x}, t)=\sum_{i} h_{i}(\mathbf{x}, t)
$$

\subsection{Boundary conditions}

Boundary treatment is one of the key issues in LBM modelling interfacial flows. In a typical simulation of thermocapillary flow in a microchannel there are three types of boundary conditions to be resolved: fluid flow boundary condition, thermal boundary condition, and wetting boundary condition. As we know, the halfway bounce-back has been widely used to handle complicated flow boundary conditions, e.g. in porous media, for its ease in implementation. As shown in Fig. 1, the link between the solid node $\mathbf{x}_{s}$ and the fluid node $\mathbf{x}_{f}$ intersects the physical boundary at $\mathbf{x}_{w}$, which is located at the midpoint of the link. The relationship between $\mathbf{x}_{s}$ and $\mathbf{x}_{f}$ is $\mathbf{x}_{s}=\mathbf{x}_{f}+\mathbf{e}_{i} \delta_{t}$. Following Ladd's halfway bounce-back scheme [79], the particle distribution function entering from "inside" the solid $\varphi_{i}\left(\mathbf{x}_{f}, t+\delta_{t}\right)$ (with $\varphi_{i}=f_{i}$ or $g_{i}$ ) is determined by

$$
\varphi_{\bar{i}}\left(\mathbf{x}_{f}, t+\delta_{t}\right)=\varphi_{i}^{+}\left(\mathbf{x}_{f}, t\right)-2 w_{i} \varrho\left(\mathbf{x}_{f}\right) \frac{\mathbf{e}_{i} \cdot \mathbf{u}_{w}}{c_{s}^{2}}
$$

for a moving wall with velocity $\mathbf{u}_{w}$, where $\mathbf{e}_{i}=-\mathbf{e}_{i}$; the superscript ' + ' denotes the post-collision, pre-streaming value of the particle distribution function; $\varrho$ is taken as $\rho$ (or $\phi$ ) for $\varphi_{i}=f_{i}$ (or $g_{i}$ ). This boundary condition can prevent the boundary "mass leakage", which is indispensable for a flow with small velocity.

The thermal boundary conditions are imposed by a general halfway bounce-back scheme recently proposed by Zhang et al. [61]. For the Dirichlet boundary condition, the particle distribution function entering from the inside of the solid is calculated as

$$
h_{\bar{i}}\left(\mathbf{x}_{f}, t+\delta_{t}\right)=-h_{i}^{+}\left(\mathbf{x}_{f}, t\right)+2 w_{i} T_{w}\left[1+\frac{\left(\mathbf{e}_{i} \cdot \mathbf{u}_{w}\right)^{2}}{2 c_{s}^{4}}-\frac{\left|\mathbf{u}_{w}\right|^{2}}{2 c_{s}^{2}}\right],
$$

where $T_{w}$ is the temperature at the boundary node $\mathbf{x}_{w}$. For the adiabatic boundary condition, the boundary temperature $T_{w}$ can be obtained by solving equation $\mathbf{n}_{w} \cdot \nabla T=0$ using a finite-difference approximation:

$$
\mathbf{n}_{w} \cdot \nabla T=\frac{T_{w}-T_{f}}{0.5 \mathbf{n}_{w} \cdot \mathbf{e}_{i} \delta_{x}}=0,
$$

i.e., $T_{w}=T_{f}$, where $T_{f}$ is the temperature at the fluid node neighboring the physical boundary, and $\mathbf{e}_{i}$ is the particular lattice velocity associated with the link that crosses the boundary. Once the boundary temperature $T_{w}$ is obtained, the unknown distribution $h_{\bar{i}}\left(\mathbf{x}_{f}, t+\delta_{t}\right)$ can be calculated through Eq. (45) similar to the Dirichlet boundary condition. In implementing the wetting boundary condition (specially, to enforce the natural boundary condition, Eq. (5)), we follow the way of Braint et al. [80]. 


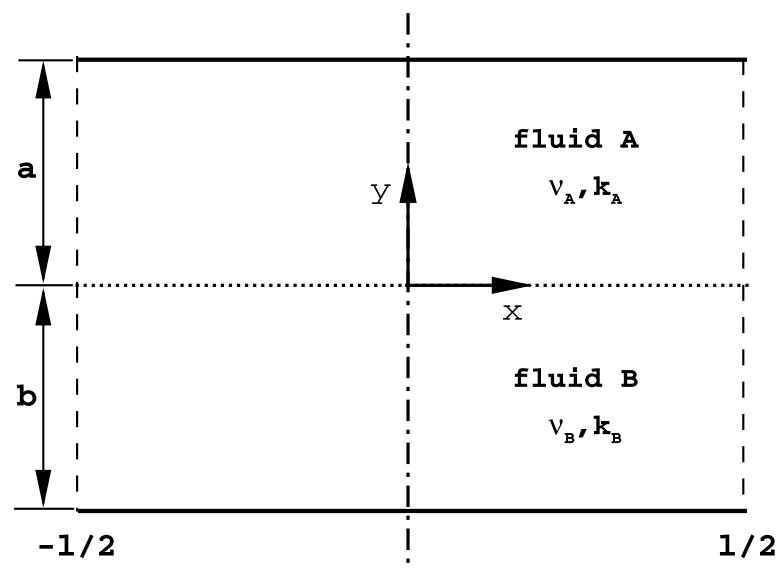

Fig. 2. The geometric setup depicting two immiscible fluids in a microchannel. The temperatures of the lower and upper walls are $T(x,-b)=T_{h}+$ $T_{0} \cos (\omega x)$ and $T(x, a)=T_{c}$, respectively, where $T_{h}>T_{c}>T_{0}$ and $\omega=\frac{2 \pi}{l}$ is a wave number.

\section{Results and discussion}

Thermocapillary motion of a droplet can be characterized by several important dimensionless parameters, including Reynolds number (Re), Marangoni number (Ma), capillary number (Ca), fluid viscosity ratio $(\lambda)$, and thermal conductivity ratio $(\chi)$. Without losing generality, we choose the fluid B as the continuous phase in this study, so these dimensionless parameters are defined as follows:

$$
\operatorname{Re}=\frac{L U}{v_{B}}, \quad \mathrm{Ma}=\frac{L U}{k_{B}}=\operatorname{Re} \cdot \operatorname{Pr}, \quad \mathrm{Ca}=\frac{U \eta_{B}}{\sigma_{\text {ref }}}, \quad \lambda=\frac{\eta_{A}}{\eta_{B}}, \quad \chi=\frac{k_{A}}{k_{B}},
$$

where $L$ and $U$ are the characteristic length and velocity of the system, respectively, and $\operatorname{Pr}$ is the Prandtl number.

\subsection{Model validation}

To verify the proposed phase-field LBM, we first simulate the thermocapillary-driven flow in a heated microchannel with two superimposed planar fluids [81]. Note that the wall wettability is not taken into account in this problem. The setup of the problem is shown in Fig. 2. The heights of the upper fluid A and the lower fluid B are $a$ and $b$, respectively, while the fluids extend to infinity in the $x$-direction. We impose a uniform temperature to the upper wall and a sinusoidal temperature (which is higher than that of the upper wall) to the lower wall as

$$
T(x, a)=T_{c},
$$

and

$$
T(x,-b)=T_{h}+T_{0} \cos (\omega x),
$$

respectively, where $0<T_{0}<T_{c}<T_{h}$, and $\omega=\frac{2 \pi}{l}$ is a wave number with $l$ being a length scale. The above thermal boundary conditions establish a periodic temperature field in the $x$-direction with a period length of $l$. Therefore, it is sufficient to only consider the solution in one period domain with $-\frac{l}{2} \leqslant x<\frac{l}{2}$. Re, Ma and Ca can be easily calculated through Eq. (47) with the characteristic length $L=b$ and the characteristic velocity $U=\frac{\left|\sigma_{T}\right| T_{0}}{l} \frac{b}{\eta_{B}}$. When these dimensionless numbers become negligibly small, the interface is thought to remain flat, and the momentum and energy equations can be simplified to be linear. By solving the simplified governing equations with the stress boundary condition (Eq. (9)) at the interface, the temperature and velocity fields can be analytically obtained and are given by [81]

$$
\begin{aligned}
T(x, y)= & \frac{\left(T_{c}-T_{h}\right) y+\chi T_{c} b+T_{h} a}{a+\chi b}+T_{0} f(\tilde{a}, \tilde{b}, \chi) \sinh (\tilde{a}-\omega y) \cos (\omega x), \\
u_{x}(x, y)= & U_{\max }\left\{\left[C_{1}^{a}+\omega\left(C_{2}^{a}+C_{3}^{a} y\right)\right] \cosh (\omega y)\right. \\
& \left.+\left(C_{3}^{a}+\omega C_{1}^{a} y\right) \sinh (\omega y)\right\} \sin (\omega x), \\
u_{y}(x, y)= & -\chi U_{\max }\left[C_{1}^{a} y \cosh (\omega y)+\left(C_{2}^{a}+C_{3}^{a} y\right) \sinh (\omega y)\right] \cos (\omega x),
\end{aligned}
$$

in the upper fluid $A$ and 


$$
\begin{aligned}
T(x, y)= & \frac{\chi\left(T_{c}-T_{h}\right) y+\chi T_{c} b+T_{h} a}{a+\chi b} \\
& +T_{0} f(\tilde{a}, \tilde{b}, \chi)[\sinh (\tilde{a}) \cosh (\omega \tilde{y})-\chi \sinh (\omega y) \cosh (\tilde{a})] \cos (\omega x), \\
u_{x}(x, y)= & U_{\max }\left\{\left[C_{1}^{b}+\omega\left(C_{2}^{b}+C_{3}^{b} y\right)\right] \cosh (\omega y)\right. \\
& \left.+\left(C_{3}^{b}+\omega C_{1}^{b} y\right) \sinh (\omega y)\right\} \sin (\omega x), \\
u_{y}(x, y)= & -\chi U_{\max }\left[C_{1}^{b} y \cosh (\omega y)+\left(C_{2}^{b}+C_{3}^{b} y\right) \sinh (\omega y)\right] \cos (\omega x),
\end{aligned}
$$

in the lower fluid B. In Eqs. (50)-(55), the unknown constants are determined by

$$
\begin{aligned}
& \tilde{a}=a \omega ; \quad \tilde{b}=b \omega, \\
& f(\tilde{a}, \tilde{b}, \chi)=[\chi \sinh (\tilde{b}) \cosh (\tilde{a})+\sinh (\tilde{a}) \cosh (\tilde{b})]^{-1}, \\
& C_{1}^{a}=\frac{\sinh ^{2}(\tilde{a})}{\sinh ^{2}(\tilde{a})-\tilde{a}^{2}} ; \quad C_{2}^{a}=\frac{-a \tilde{a}}{\sinh ^{2}(\tilde{a})-\tilde{a}^{2}} ; \quad C_{3}^{a}=\frac{2 \tilde{a}-\sinh (2 \tilde{a})}{2\left[\sinh ^{2}(\tilde{a})-\tilde{a}^{2}\right]}, \\
& C_{1}^{b}=\frac{\sinh ^{2}(\tilde{b})}{\sinh ^{2}(\tilde{b})-\tilde{b}^{2}} ; \quad C_{2}^{b}=\frac{-b \tilde{b}}{\sinh ^{2}(\tilde{b})-\tilde{b}^{2}} ; \quad C_{3}^{b}=\frac{\sinh (2 \tilde{b})-2 \tilde{b}}{2\left[\sinh ^{2}(\tilde{b})-\tilde{b}^{2}\right]},
\end{aligned}
$$

and

$$
U_{\max }=-\left(\frac{T_{0} \sigma_{T}}{\eta_{2}}\right) g(\tilde{a}, \tilde{b}, \chi) h(\tilde{a}, \tilde{b}, \lambda),
$$

where

$$
g(\tilde{a}, \tilde{b}, \chi)=\sinh f(\tilde{a}, \tilde{b}, \chi),
$$

and

$$
h(\tilde{a}, \tilde{b}, \lambda)=\frac{\left[\sinh ^{2}(\tilde{a})-\tilde{a}^{2}\right]\left[\sinh ^{2}(\tilde{b})-\tilde{b}^{2}\right]}{\lambda\left[\sinh ^{2}(\tilde{b})-\tilde{b}^{2}\right][\sinh (2 \tilde{a})-2 \tilde{a}]+\left[\sinh ^{2}(\tilde{a})-\tilde{a}^{2}\right][\sinh (2 \tilde{b})-2 \tilde{b}]} .
$$

Numerical simulations are carried out in a $200 \times 102$ lattice domain with the initial heights of fluid layer $a=b=50$ lattices. Periodic boundary conditions are applied on the left and right boundaries of the domain. On the upper and lower walls, no-slip boundary conditions are imposed, and the wall temperatures are specified through Eqs. (48) and (49), where $T_{h}=20, T_{c}=10$ and $T_{0}=4$. The fluid properties and numerical parameters are chosen as $\sigma_{T}=-5 \times 10^{-4}, T_{\text {ref }}=10$, $\sigma_{\text {ref }}=2.5 \times 10^{-2}, \eta_{A, B}=0.25, k_{B}=0.2, \epsilon=1.0$ and $M_{\phi}=5 \times 10^{-2}$. These values result in Re, Ma and Ca of typical value of $O(0.01)$ or at most $O(0.1)$. To show the effect of thermal conductivity ratio on the induced flow and temperature field, both $k_{A}=0.2$ and $k_{A}=0.02$ are simulated such that $\chi=1$ and $\chi=1 / 10$.

Fig. 3 shows equispaced contours of temperature field for the thermal conductivity ratio: (a) $\chi=1$ and (b) $\chi=1 / 10$. It can be clearly seen that our numerical results (solid contours) agree well with the analytical solutions (dashed contours) as given by Eqs. (50) and (53). This suggests that the halfway bounce-back scheme Eq. (45) is able to enforce accurately the thermal boundary condition with non-uniform wall temperature. Note that our simulated isotherms slightly deviate from the analytical ones in the neighborhood of the interface for $\chi=1 / 10$, which is caused by the finite interface thickness of the phase-field model and the jump of thermal conductivity across the interface. At a low thermal conductivity ratio, i.e. $\chi=1 / 10$, the isotherms approaching from the lower fluid tend to be normal to the interface, implying that heat transfer between the lower fluid and the interface in the $y$-direction is close to zero. Fig. 4 shows the comparison of velocity vectors between our simulation results and the analytical solutions as given by Eqs. (51), (52), (54), and (55) for $\chi=1$ and $1 / 10$. The simulated velocity vectors also agree well with the analytical solutions except those close to the interface at $\chi=1 / 10$, consistent with the deviation in isotherms. Finally, it can be found by comparing Fig. 4(a) with Fig. 4(b) that the thermocapillary-driven convection will be strengthened with decreasing thermal conductivity ratio.

To assess the fluid-solid interaction model given by Eq. (5) implemented in the proposed phase-field model, we perform static contact angle simulations in a $300 \times 100$ lattice domain. The dependence of the interfacial tension on the temperature is switched off so that a constant interfacial tension is used. The initial condition is a semi-circular stationary droplet (fluid A) sitting along the center line on the bottom wall. The top wall is assumed to be neutral wetting, i.e., $\theta^{e q}=90^{\circ}$. The periodic boundary condition is used in the horizontal direction while the halfway bounce-back boundary condition, i.e. Eq. (45), is imposed at top and bottom in the vertical direction. The relaxation parameters are fixed at $\tau_{A}=3.0$ and $\tau_{B}=0.7$, which gives a viscosity ratio of $\lambda=\left(\tau_{A}-0.5\right) /\left(\tau_{B}-0.5\right)=12.5$. Other parameters are chosen as: $\sigma=0.038, \epsilon=\sqrt{2}$ and $M_{\phi}=0.01$. We run the simulation until the shape of droplet does not change, i.e. reaching an equilibrium state. Different contact angles can be achieved through adjusting the dimensionless wetting potential $\Omega_{c}$ according to Eq. (5). Fig. 5(a)-(c) shows equilibrium shapes of the droplet with the wetting potential $\Omega_{c}=-0.5,0$ and 0.5 , respectively. Their corresponding equilibrium contact angles, calculated from the measured droplet height and base diameter, are $60.22^{\circ}, 89.97^{\circ}$ and 
(a)

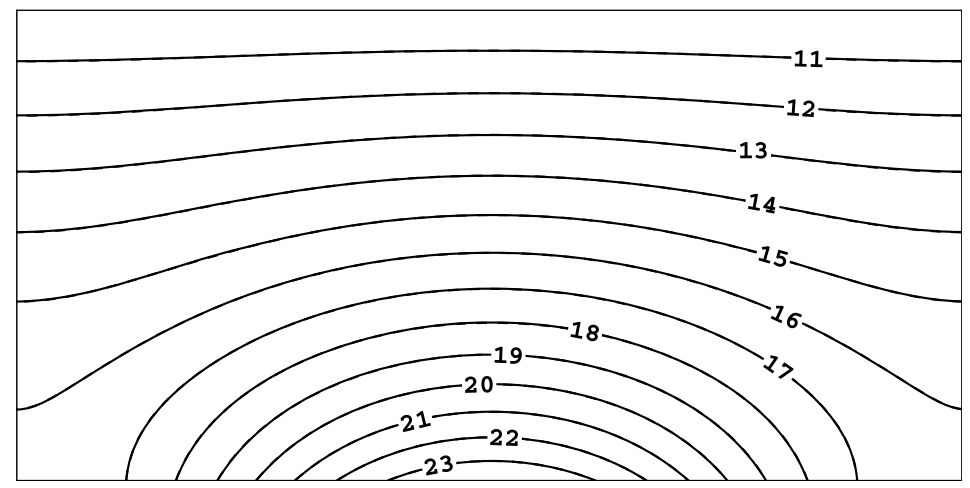

(b)

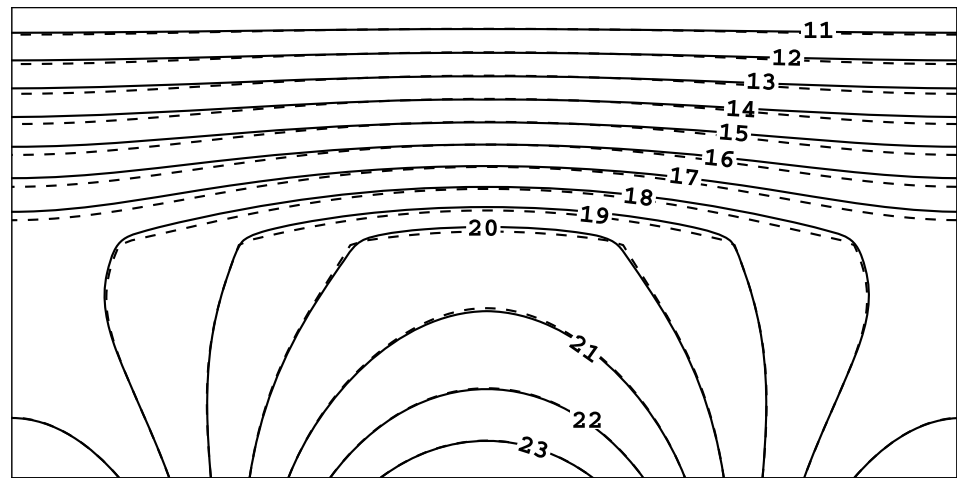

Fig. 3. Temperature contours for fluid systems with thermal conductivity ratios of (a) $\chi=1$ and (b) $\chi=1 / 10$. Analytical solutions and simulation results are represented by the dashed lines and solid lines, respectively, labelled with temperature values.

$119.54^{\circ}$, respectively. The simulated equilibrium contact angle as a function of the dimensionless wetting potential for a wider range is presented in Fig. 6. It is clearly observed that the simulated results by the present LBM agree well with the theoretical solution, Eq. (3), in the range of contact angle from $30^{\circ}$ to $150^{\circ}$. Minimization of unphysical spurious velocities at the interface still remains a challenge in many multiphase models. Fig. 7 shows the time evolution of the maximum spurious velocities $\left(U_{\max }\right)$ for various contact angles. For all of the contact angles under consideration, the maximum spurious velocities in the steady state range from $2 \times 10^{-7}$ to $2.42 \times 10^{-6}$, which are much smaller than those obtained by other multiphase LBM models $[37,42,46,32,60,41]$. Therefore, the present phase-field model is well suited for simulating multiphase flows in confined microfluidic geometries. In addition, it is interesting to make some remarks concerning the choice of free parameters $s_{1}, s_{2}$ and $s_{4}$ in the diagonal relaxation matrix $\mathbf{S}$. First of all, the free parameters are not uniquely determined although Lallemand and Luo [73] provided some guidelines to choose some of them. It is therefore not surprising that a number of forms of diagonal relaxation matrix have been reported in literature with different free parameters, e.g., Refs. [71,82-84,59,85-87]. To date, trial-and-error approach is still required to find the most stable and reliable set of free parameters for a specific problem. Secondly, it is acknowledged that the free parameters can affect the magnitude of spurious velocities in multiphase/multicomponent LBM models $[32,59,60]$ though they are not associated with the hydrodynamic moments. For the simulations of static contact angle, we have found that the spurious velocities obtained with our choice of $s_{1}=1.63, s_{2}=1.14$ and $s_{4}=1.92$ are smaller than those obtained by the well-known Two-Relaxation-Time (TRT) algorithm $\left(s_{1}=s_{2}=\frac{1}{\tau_{f}}\right.$ and $\left.s_{4}=\frac{8\left(2-s_{1}\right)}{8-s_{1}}\right)$ [88,75], the MRT of Pooley et al. $\left(s_{1}=s_{2}=s_{4}=1\right)$ [32], and the MRT of Fakhari and Lee [87]. For example, in the case of $\Omega_{c}=\frac{\sqrt{3}}{2}$ the magnitudes of maximum spurious velocity are $2.42 \times 10^{-6}$ for our choice, $4.89 \times 10^{-6}$ for the TRT, $6.73 \times 10^{-6}$ for the MRT of Pooley et al., and $3.69 \times 10^{-6}$ for the MRT of Fakhari and Lee, respectively. Finally, the MRT algorithm should be more stable than the BGK model $[73,88,74,75,32,59,60]$ even if the choice of free parameters is not optimal. This is also demonstrated by our simulations, in which the fluid viscosities are decreased to a very low value, e.g. $\tau_{A}=0.53$ and $\tau_{B}=0.5024$, while the other parameters are kept the same as the above. As can be seen in Fig. 8, the droplet will be driven away from the bottom wall by the strong spurious velocities when the BGK model is used, which is unphysical. However, our MRT algorithm is able to produce the correct equilibrium contact angle, with the predicted value of $151.5^{\circ}$ in good agreement with its theoretical value of $150^{\circ}$. Based on the limited numerical comparisons shown here, the guidelines given by Lallemand and Luo [73] (recall that our free parameters are chosen following their recommendation) seem to be applicable as well in the present multiphase MRT model. However, further study is required to provide more insights in determining optimal parameters. 
(a)

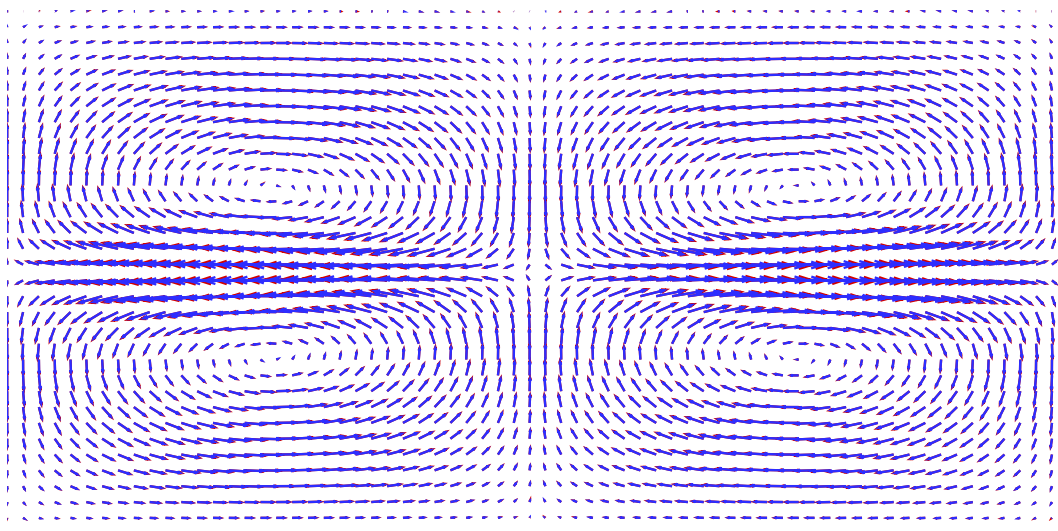

(b)

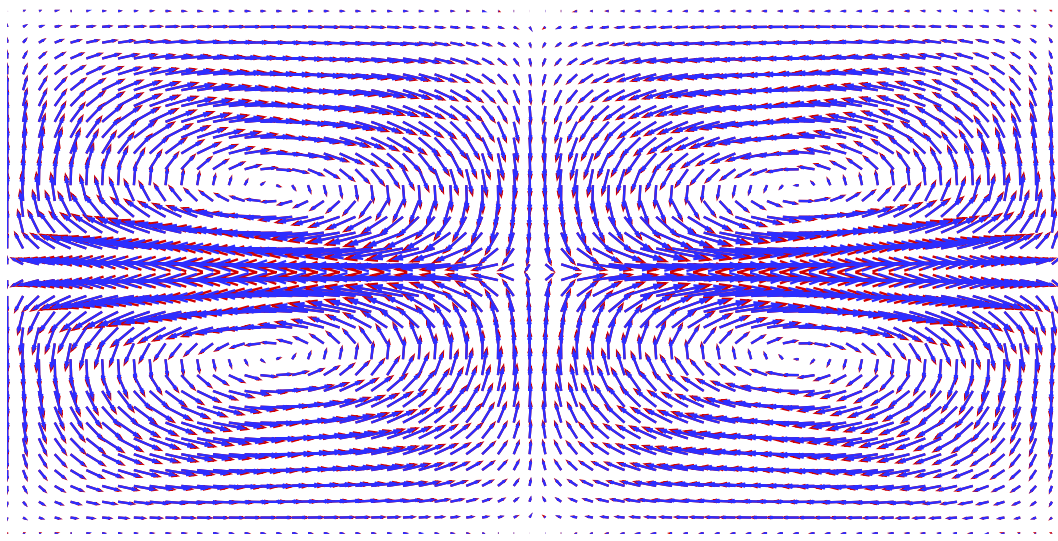

Fig. 4. (Color online.) Velocity vectors for fluid systems with thermal conductivity ratios of (a) $\chi=1$ and (b) $\chi=1 / 10$. Velocity vectors are shown at every third grid point. Analytical solutions and simulation results are represented by the red and blue lines with arrow, respectively.

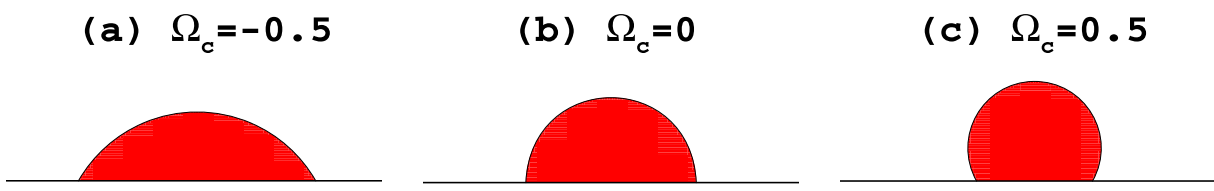

Fig. 5. (Color online.) Droplet on a solid surface with the dimensionless wetting potential of (a) $\Omega_{c}=-0.5$, (b) $\Omega_{c}=0$ and (c) $\Omega_{c}=0.5$. The corresponding contact angles are $60.22^{\circ}, 89.97^{\circ}$ and $119.54^{\circ}$, respectively.

\subsection{Thermocapillary manipulation of a moving microdroplet on a solid substrate}

Historically, the research on thermocapillary flow mainly focused on immersed droplets in an infinite fluid medium under the microgravity conditions where no solid boundary is involved, avoiding complex fluid-surface interactions. Since interfacial tension plays a dominant role compared to other forces for microscale droplets, microfluidics has opened up a new avenue for droplet manipulation by the use of optically-induced thermocapillary convection, i.e., the so-called optocapillarity. Different from the flows in an infinite domain, the confinement and wettability of channel walls would quantitatively or qualitatively modify the physics of thermocapillary migration in a microfluidic channel $[89,90]$. Although a color-fluid LB model [53] has been developed to simulate optocapillary flows in a microchannel, and 'blocking' behavior of droplet was reproduced successfully, the moving droplet is only allowed to be fully immersed in the carrier fluid without consideration of wettability of channel walls. Thus, this model is inapplicable in most microfluidic environments where disperse droplets interact directly with channel walls. In addition, the droplet dynamics in optocapillary flows is still poorly understood especially when the wall wettability is taken into account. Here we will apply the proposed phase-field LBM to numerically study thermocapillary flows for a droplet adhering on a solid wall and subject to a simple shear flow in a microchannel, in which a laser is used to locally heat the fluid. The effect of wall wettability and fluid viscosity ratio on droplet dynamic behavior will be investigated for a constant thermal conductivity ratio. For the sake of simplicity, here we approximate the heating from the laser as a local heat source of a Gaussian form: 


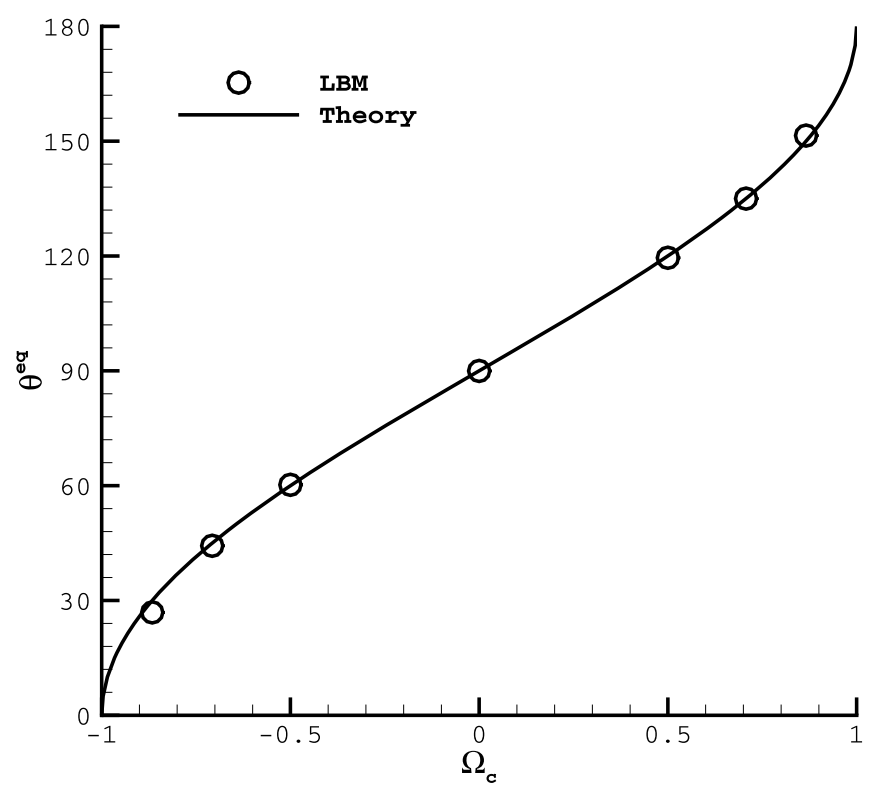

Fig. 6. Contact angle as a function of the dimensionless wetting potential with the simulation results represented by discrete points and the solid line from the theoretical predictions by Eq. (3).

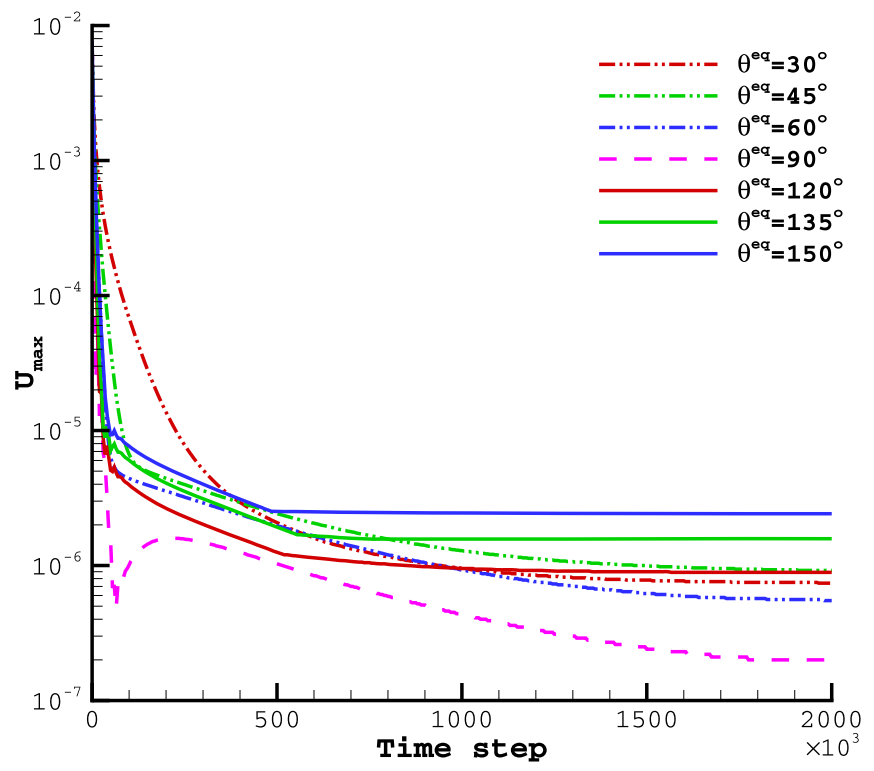

Fig. 7. (Color online.) Time evolution of the maximum spurious velocities $\left(U_{\max }\right)$ at various contact angles.

$$
q_{T}= \begin{cases}Q_{S} \mathrm{e}^{-2 \frac{\left(x-x_{S}\right)^{2}+\left(y-y_{s}\right)^{2}}{w_{s}^{2}}}, & \text { if }\left[\left(x-x_{s}\right)^{2}+\left(y-y_{s}\right)^{2}\right] \leqslant d_{s}^{2}, \\ 0, & \text { otherwise, }\end{cases}
$$

where $Q_{s}$ is the maximum heat flux of the hot spot, $x_{s}$ and $y_{s}$ are the position of hot spot where the heat flux is taken to be of the maximum value, $d_{s}$ is the size of the diffused hot spot, and $w_{s}$ is a parameter controlling the profile of heat flux.

The detailed problem setups are given as follows. As shown in Fig. 9, a stationary semi-circular droplet of radius $R=32$ initially sits on a smooth substrate at the bottom. The computational domain size is $L_{x} \times L_{y}=8 R \times 2 R$ and the initial droplet center is $\left(x_{C}, y_{C}\right)=(65,0)$. The bottom wall is kept stationary, and the top wall moves towards the right with a constant velocity $U_{w}$, yielding a constant shear rate $\gamma=U_{w} / L_{y}$. Besides, a constant temperature $T=0$ is specified at the bottom and top walls. In the horizontal directions, periodic boundary conditions are applied for fluid flows while adiabatic boundary conditions are used for temperature. The fluid is locally heated by a laser lighting source with its distribution given by Eq. (62), in which $x_{s}=181, y_{s}=21, w_{s}=6.0, d_{s}=8.0$, and $Q_{s}=0.2$. To reproduce the thermocapillary blocking, 


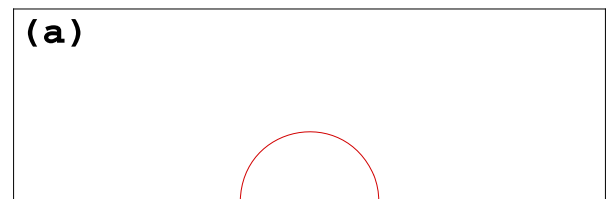

(c)

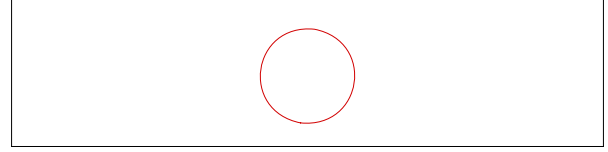

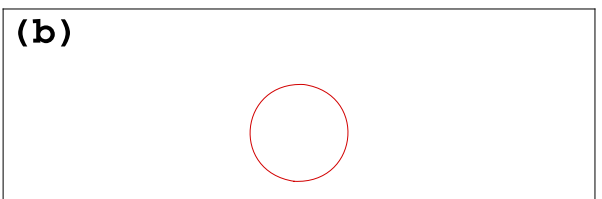

(d)

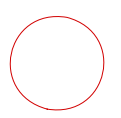

Fig. 8. (Color online.) Snapshots of a droplet, which is initially placed on the bottom wall with a semi-circular shape for $\tau_{A}=0.53$, $\tau_{B}=0.5024$ and $\Omega_{c}=\frac{\sqrt{3}}{2}$, taken at times (a) $t=0$, (b) $t=20,000$, (c) $t=500,000$, and (d) $t=1,000,000$. Note that the BGK model is used in the simulation. The red solid lines are zero contours of the order parameter $\phi$.

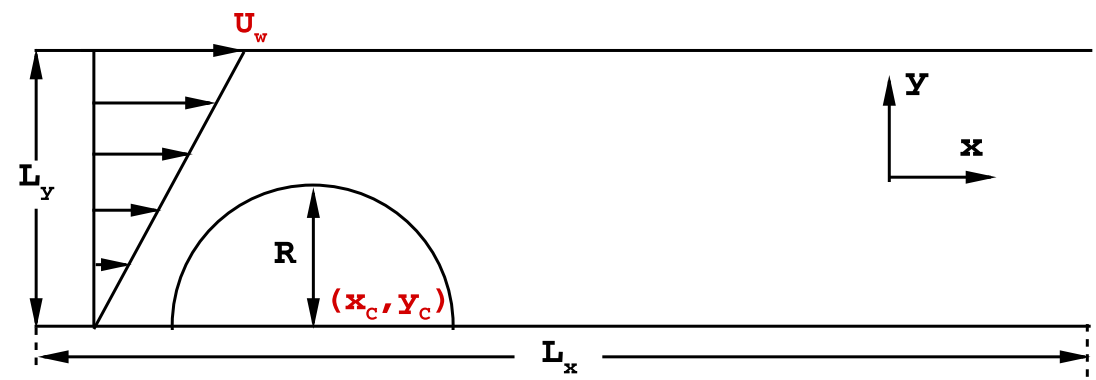

Fig. 9. A schematic illustration of the geometry setup for simulating a moving droplet driven by a simple shear flow subjected to a laser heating. A semicircular droplet is initially placed on the bottom substrate with its center located at $\left(x_{C}, y_{C}\right)$.

the coefficient of interfacial tension variation with temperature, $\sigma_{T}$, should be positive $[27,28]$. Note that in our study an "effective" coefficient is directly used accounting for both temperature and concentration effects in the presence of surfactant. Specifically, we take $\sigma_{T}=2 \times 10^{-4}$, and $\sigma_{\text {ref }}=5 \times 10^{-3}$ at the reference temperature $T_{\text {ref }}=0$. The dimensionless numbers $\mathrm{Ca}, \mathrm{Re}, \mathrm{Ma}$, and $\chi$ are respectively fixed at $0.01,0.16,0.08$, and 1 , where the characteristic length and velocity are defined by $L=R$ and $U=\gamma R$. In addition, the Cahn number can be calculated as $C n=\epsilon / R \simeq 0.044$, and the Peclet number $\mathrm{Pe}=\gamma R \epsilon / M$ is of $\mathcal{O}(1)$, which is required to capture correct droplet dynamical behavior [91,92].

The contact angle plays an important role in determining the dynamical behavior of a moving droplet in a microchannel due to large surface-to-volume ratio. The effect of contact angle is first investigated for a fixed fluid pair with $\lambda=1$. Different contact angles are achieved through adjusting the value of $\Omega_{c}$ while keeping the other parameters fixed. When a laser heating source is applied, it is observed that the droplet motion can be blocked to some extent, which depends on the magnitude of the contact angle. As $\theta^{\text {eq }}$ increases from $45^{\circ}$ to $135^{\circ}$, the droplet motion can undergo two states. At the smallest $\theta^{e q}$, the droplet motion is partially blocked. When $\theta^{e q}$ increases beyond a critical value $\theta_{c}^{e q}\left(45^{\circ}<\theta_{c}^{e q}<60^{\circ}\right)$, the droplet motion is completely blocked, so the droplet finally rests at a site adjacent to the center $\left(x_{s}, y_{s}\right)$ of the heating source. Fig. 10 shows the time evolution of the droplet shape, position, and the streamlines around the moving droplet for $\theta^{e q}=45^{\circ}$, which corresponds to the state that the droplet motion is partially blocked. The dimensionless time is defined by the shear rate as $t^{*}=\gamma t$. At an early time, i.e., $t^{*}=6.25$, since the droplet is far away from the laser heating source and the viscous force driving the droplet motion is small, the streamlines are almost straight. As the droplet moves towards the heating source, a small vortex begins to grow at the substrate inside the droplet (see Fig. $10(\mathrm{~b})$ ). At $t^{*}=18.75$, the droplet moves further towards the heating source. The size of vortex inside the droplet increases, and a small vortex (i.e., the external vortex) starts to appear in the carrier fluid and close to the front end of the droplet. Shortly, another small vortex starts to arise near the external vortex. But different from the external vortex, it is located on the other side of the droplet interface with an opposite direction of rotation (see Fig. 10(d)). As the time elapses, the internal main vortex first grows slightly and then diminishes gradually with the largest size occurring at $t^{*}=28.13$, while the other two vortices both grow until $t^{*}=50.0$ (see Fig. $10(\mathrm{e})-(\mathrm{g})$ ). Subsequently, the droplet keeps moving towards the right, and all the vortices decrease significantly in size (see Fig. $10(\mathrm{~h})-(\mathrm{j})$ ). At $t^{*}=59.69$, we can notice that the external vortex has vanished and its neighboring internal vortex also reduces to a small size. As the droplet passes through the center of heating source, the neighboring internal vortex does not decrease further but increases (see Fig. 10(k) and (l)). This increase attributes to the Marangoni convection at the top interface of the droplet, where a large temperature gradient may happen due to the localized heating from laser. However, the internal main vortex continues to decrease and even becomes non-dominant in size (see Fig. 10(1)) (note that we still call it main vortex in order to avoid inconsistency). 


\section{(a)}

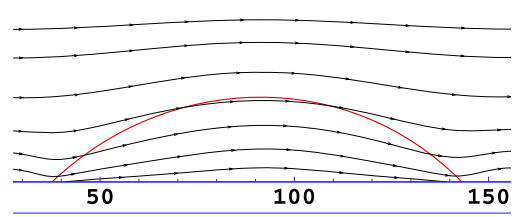

(d)

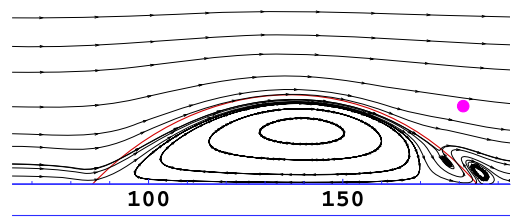

(g)

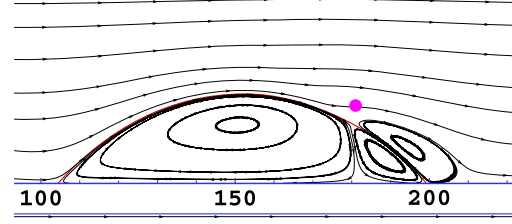

(j)

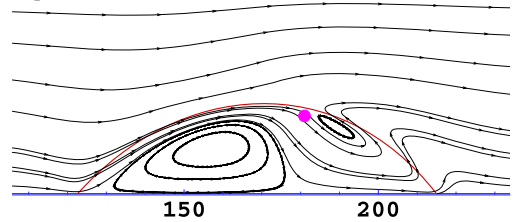

(b)

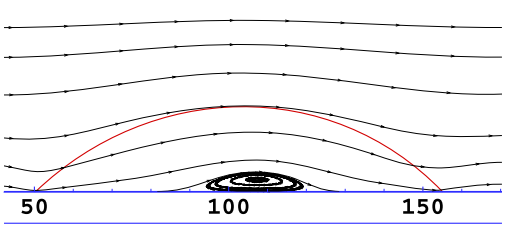

(e)

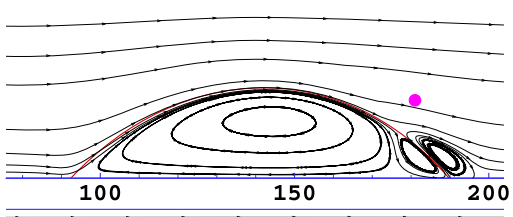

(h)

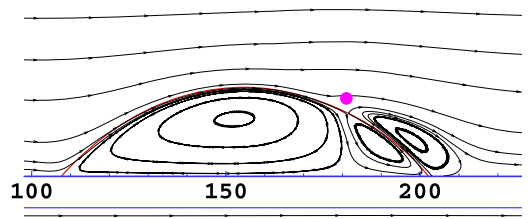

(k)

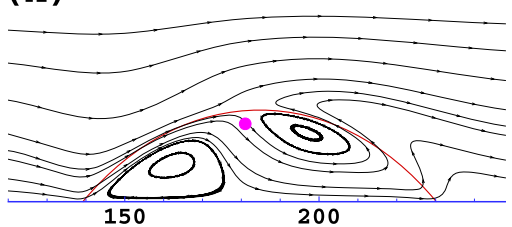

(c)

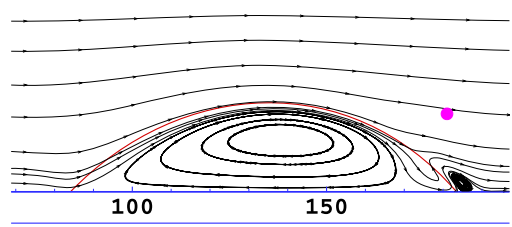

(f)

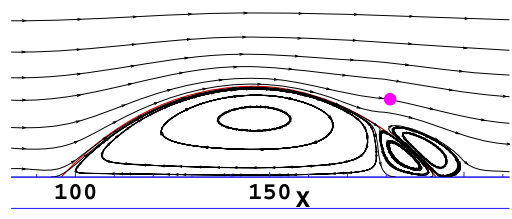

(i)

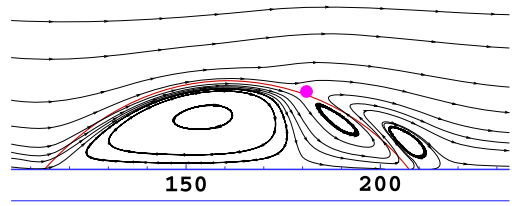

(1)

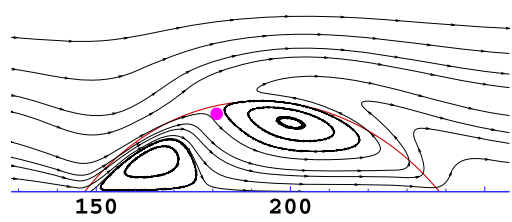

Fig. 10. (Color online.) The time evolution of droplet shape, position and streamlines surrounding the droplet for $\theta^{e q}=45^{\circ}$ with the times taken as: (a) $t^{*}=6.25$, (b) $t^{*}=9.38$, (c) $t^{*}=18.75$, (d) $t^{*}=19.38$, (e) $t^{*}=23.44$, (f) $t^{*}=28.13$, (g) $t^{*}=50.0$, (h) $t^{*}=54.69$, (i) $t^{*}=57.81$, (j) $t^{*}=59.69$, (k) $t^{*}=62.5$, and (l) $t^{*}=64.06$. The red solid lines are zero contours of the order parameter $\phi$, the black lines with arrows are the streamlines, and the pink point is the center of the heating source.

Fig. 11 shows the time evolution of droplet shape, position, and streamline patterns around the moving droplet for $\theta^{\text {eq }}=$ $90^{\circ}$, where the droplet motion is completely blocked due to stronger Marangoni convection arising from more "effective" interfacial areas. At $t^{*}=3.13$, the droplet is far away from the laser heating source, and thus the effect of Marangoni stresses is negligibly small. However, the streamlines are less horizontal and straight than those in the case of $\theta^{e q}=45^{\circ}$ (see Fig. 11(a)), which is attributed to an increased viscous shear force. As the droplet moves forward, a clockwise-rotating vortex, i.e., the internal main vortex, appears at the bottom wall inside the droplet at $t^{*}=9.38$. This vortex quickly grows, and meanwhile its core uplifts (see Fig. 11(c)). At $t^{*}=15.63$, some S-shaped streamlines are observed in the region near the front end of the droplet, where a small external vortex also starts to grow. The internal main vortex and the external vortex continuously increase in size and between them, a new internal vortex with anticlockwise rotation has emerged (Fig. 11(e)). At a later stage, all the vortices keep growing slowly until their sizes reaching the maximum values at $t^{*}=31.25$. Since then, the positions of the droplet and vortices, as well as their sizes and shapes remain unchanged (Fig. 11(h)), indicating that the droplet has been motionless and reached the steady state. It can be clearly seen that the two counter-rotating internal vortices completely fill the droplet and the droplet behaves like a solid obstacle. Noted that in a flow around a solid obstacle, one cannot observe vortex/recirculation outside the solid for a small Reynolds number, typically Re $<1$. However, in the present simulation the temperature gradient causes the fluid to flow away from the bottom wall regions of low interfacial tension, and thereby the external vortex is formed. The existence of these stable vortex regions is a result of the balance of various forces, including the pressure, viscous stresses, and the capillary and Marangoni forces. Similar droplet behavior and flow patterns are also observed for $\theta^{e q}=60^{\circ}, 120^{\circ}$ and $135^{\circ}$. However, the contact angle affects the inception time of each vortex, and the time at which the droplet motion is completely blocked. This can be clearly seen by comparing the simulation results of two different contact angles, e.g., $\theta^{e q}=90^{\circ}$ and $\theta^{e q}=135^{\circ}$ (see Figs. 11 and 12 ). As will be shown in Fig. 14, the droplet moves at a higher velocity for more hydrophobic surface (i.e., larger contact angle), so it will be earlier to reach the region with higher temperature gradient, and intuitively the inception of each vortex and the droplet blocking will occur earlier. But this intuition seems not true for the internal main vortex, which is formed at $t^{*}=9.38$ for $\theta^{\text {eq }}=90^{\circ}$ while delayed to $t^{*}=10.0$ for $\theta^{e q}=135^{\circ}$. The delay may be associated to the larger viscous force at more hydrophobic surface such that a larger temperature gradient is required to overcome this force for generating the vortex. At $\theta^{e q}=135^{\circ}$, 


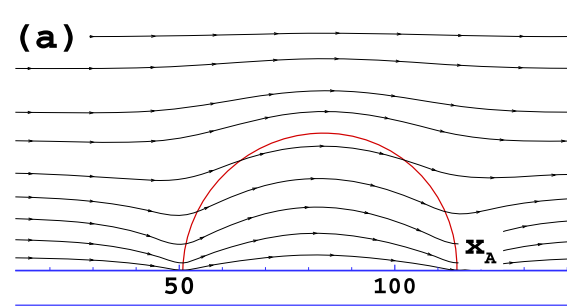

(c)
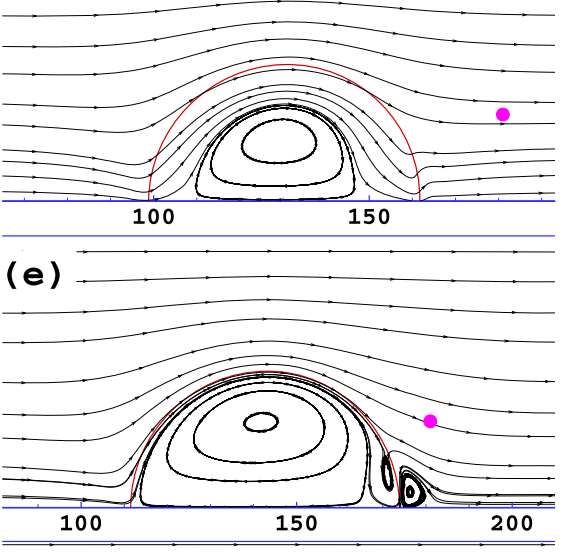

(g)

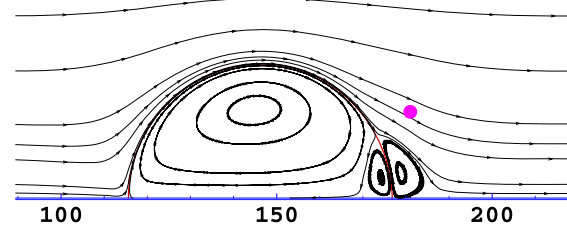

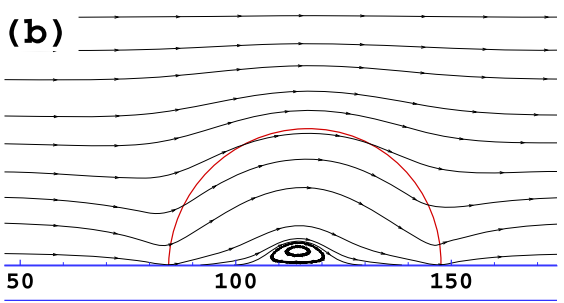

(d)

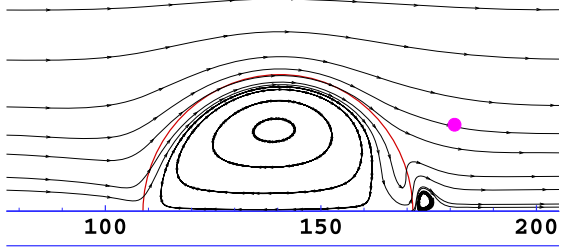

(f)

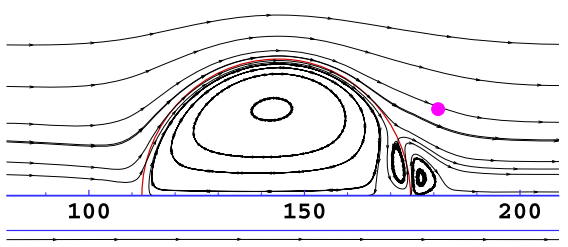

(h)

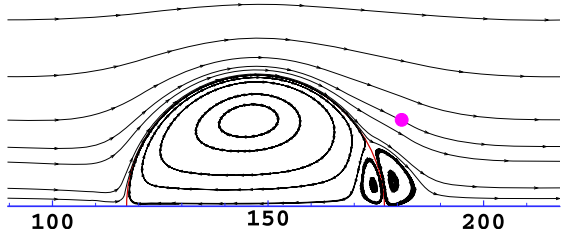

Fig. 11. (Color online.) The time evolution of droplet shape, position and streamlines surrounding the droplet for $\theta^{e q}=90^{\circ}$ with the times taken as: (a) $t^{*}=3.13$, (b) $t^{*}=9.38$, (c) $t^{*}=12.5$, (d) $t^{*}=15.63$, (e) $t^{*}=17.19$, (f) $t^{*}=17.81$, (g) $t^{*}=31.25$, and (h) $t^{*}=78.13$. The red solid lines are zero contours of the order parameter $\phi$, the black lines with arrows are the streamlines, and the pink point is the center of the heating source. Besides, $x_{A}$ in (a) denotes the $x$-position of the advancing contact point.

we also notice that the external vortex and its neighboring internal vortex are both larger in size, implying that the droplet is easier to get blocked for more hydrophobic surfaces.

Fig. 13 gives the evolution of the temperature field surrounding the moving droplet for $\theta^{e q}=120^{\circ}$ and $\lambda=1$. We can see that the temperature field has negligible change because thermal diffusion is the dominant energy transport mechanism at small Marangoni numbers. As stated previously, we use the constant Marangoni number and thermal conductivity ratio throughout this paper, i.e., $\mathrm{Ma}=0.08$ and $\chi=1$, it is therefore believed that all the temperature fields are almost the same as those given in Fig. 13 even though big differences may be observed for droplet shape and velocity field when different flow conditions are used. From the far field to the Center of Heating Source (CHS), the temperature increases, and the isotherms become denser, implying a higher temperature gradient near the CHS. However, one should notice that the temperature gradient is positive on the left side of CHS but it is negative on the right side. Considering the Marangoni force that causes the fluid to move towards the regions of high interfacial tension (or high temperature) along the interface, it is expected that the Marangoni force arising at the interface serves to block the droplet motion when the interface is located on the left side of the CHS. Once the droplet interface moves to the right side of CHS, an opposite Marangoni force, which promotes the droplet motion, will be formed at this interface. In particular, if some droplet interface is located to the left side of the CHS but the rest subject to the right side, the acceleration or deceleration of droplet will depend on which side of the interfacial force prevails. During the droplet motion, various forces are dynamically varying in magnitude and compete with each other, leading to the droplet behavior very complicated especially when the droplet motion is partially blocked. This can be clearly seen in Fig. 14, which plots the $x$-coordinate of droplet centroid as a function of the dimensionless time $t^{*}$, calculated by

$$
x_{d}(t)=\frac{\int_{V} \phi x d V}{\int_{V} \phi d V}=\frac{\sum_{\mathbf{x}} x(\mathbf{x}, t) \phi(\mathbf{x}, t)}{\sum_{\mathbf{x}} \phi(\mathbf{x}, t)}, \quad \text { where } \phi<0 .
$$



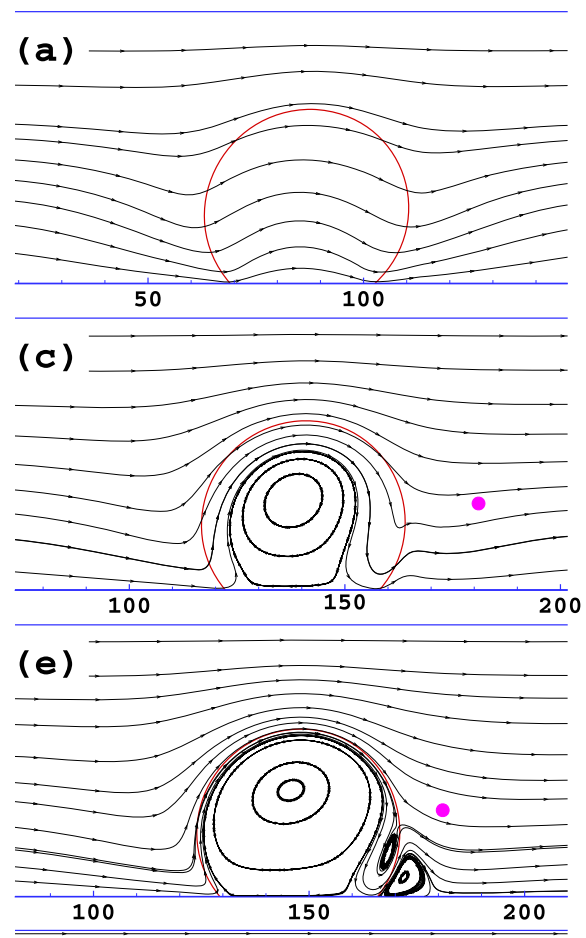

(g)

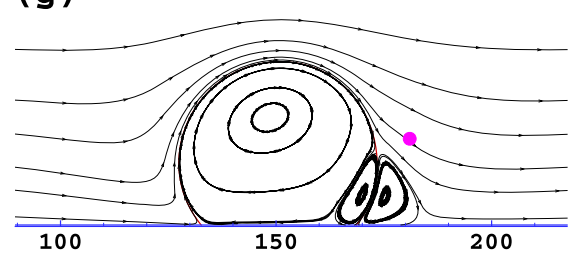

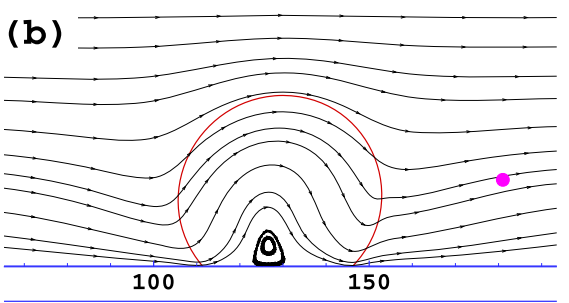
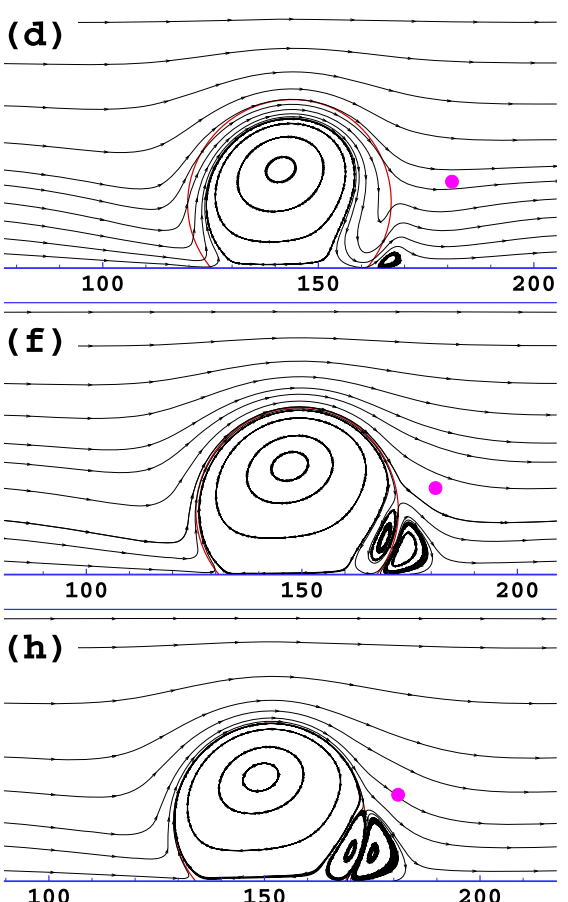

Fig. 12. (Color online.) The time evolution of droplet shape, position and streamlines surrounding the droplet for $\theta^{e q}=135^{\circ}$ with the times taken as: (a) $t^{*}=3.13$, (b) $t^{*}=10.0$, (c) $t^{*}=12.5$, (d) $t^{*}=13.44$, (e) $t^{*}=15.63$, (f) $t^{*}=17.19$, (g) $t^{*}=28.13$, and (h) $t^{*}=78.13$. The red solid lines are zero contours of the order parameter $\phi$, the black lines with arrows are the streamlines, and the pink point is the center of the heating source.

By differentiating $x_{d}$ with respect to time, we obtain that, the droplet initially slides on the substrate at a constant velocity for all the contact angles because the droplet is far from the CHS. However, the moving velocity increases with increasing contact angle. Afterwards, the droplet motion exhibits significantly different characteristics for $\theta^{e q}=45^{\circ}$ and $\theta^{e q} \geqslant 60^{\circ}$. For $\theta^{e q} \geqslant 60^{\circ}$ the droplet will continuously slows down and finally rests in the vicinity of the CHS. It can be observed in Fig. 14 that the final rest position of droplet centroid, $x_{d}(t \rightarrow \infty)$, increases as the contact angle increases. So does it mean that the droplet becomes more difficult to be blocked with an increase in $\theta^{e q}$ ? To clarify this question, we have plotted the rest position of the advancing contact point $x_{A}$ (i.e., the intersection between the droplet front and the solid surface as indicated in Fig. 11(a)) as a function of the contact angle in the inset of Fig. 14. As the contact angle increases, $x_{A}$ decreases, indicating that the droplet gets blocked more easily. Actually, the aforementioned observations that $x_{d}(t \rightarrow \infty)$ increases with increasing $\theta^{e q}$ is caused by the large horizontal length of droplet for small contact angle. For the smallest contact angle, i.e., $\theta^{e q}=45^{\circ}$, the droplet motion successively experiences the transition of constant velocity (CV), deceleration (DC), acceleration (AC), and approximately constant velocity (ACV). To better understand these droplet behaviors, we choose the discrete points $\mathrm{b}, \mathrm{d}, \mathrm{h}$ and $\mathrm{k}$ on the line of $\theta^{e q}=45^{\circ}$, which fall into the regimes of CV, DC, AC and ACV, respectively. Note that the corresponding snapshots for the four discrete points are given in Fig. 10(b), (d), (h) and (k), respectively. As we expect, the droplet is far from the CHS in the CV regime. In the DC regime, the droplet front is very close to the CHS, and the interface is mainly located on the left side of CHS. In the AC regime, the Marangoni force at the interface located on the right side of the CHS is large enough to dominate the droplet motion, where more droplet interface is exposed in the region of high temperature gradient. In the ACV regime, the droplet is passing through the CHS, and the Marangoni force at the right interface of CHS still keeps a little larger than the one at the left interface. The discrepancy in Marangoni force is quasi-balanced by the viscous stresses, and thus it is not surprising that the droplet moves at a velocity larger than the initial droplet velocity in the far field.

For a droplet/bubble migration in an infinite medium, the viscosity ratio was found to strongly affect its migration velocity [93]. It is believed that the influence of viscosity ratio is more complicated in a confined microchannel due to the 

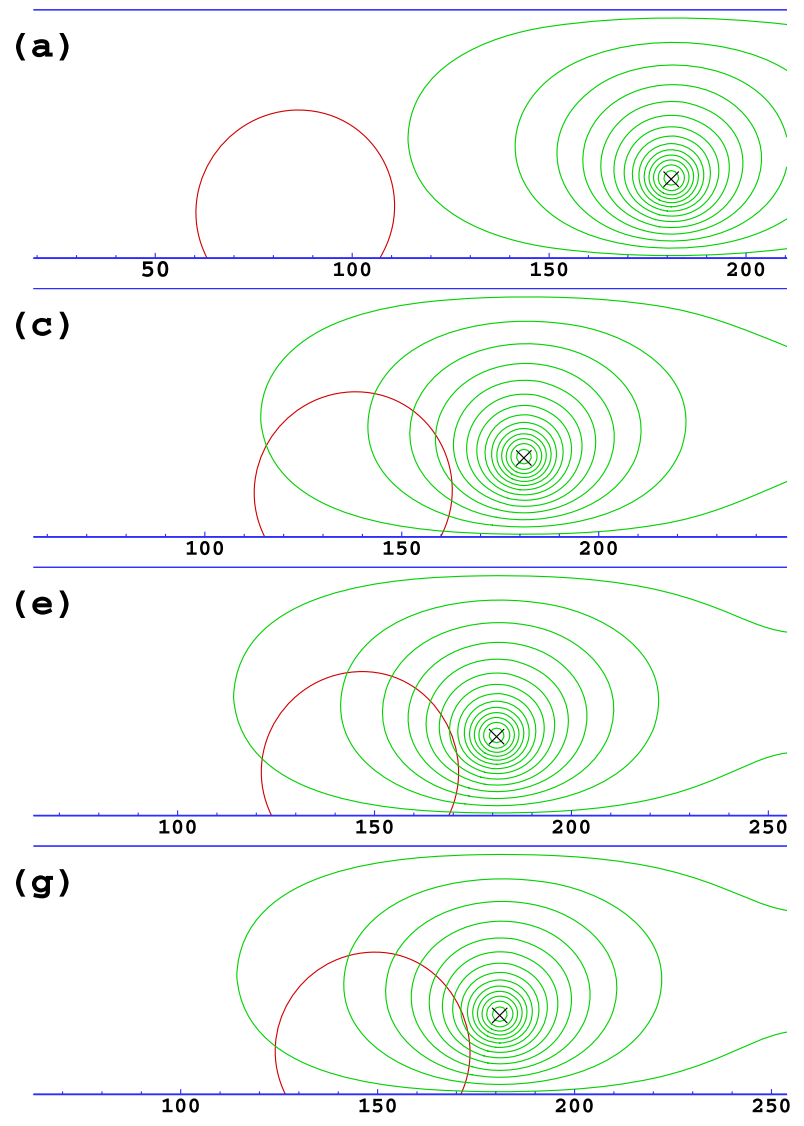
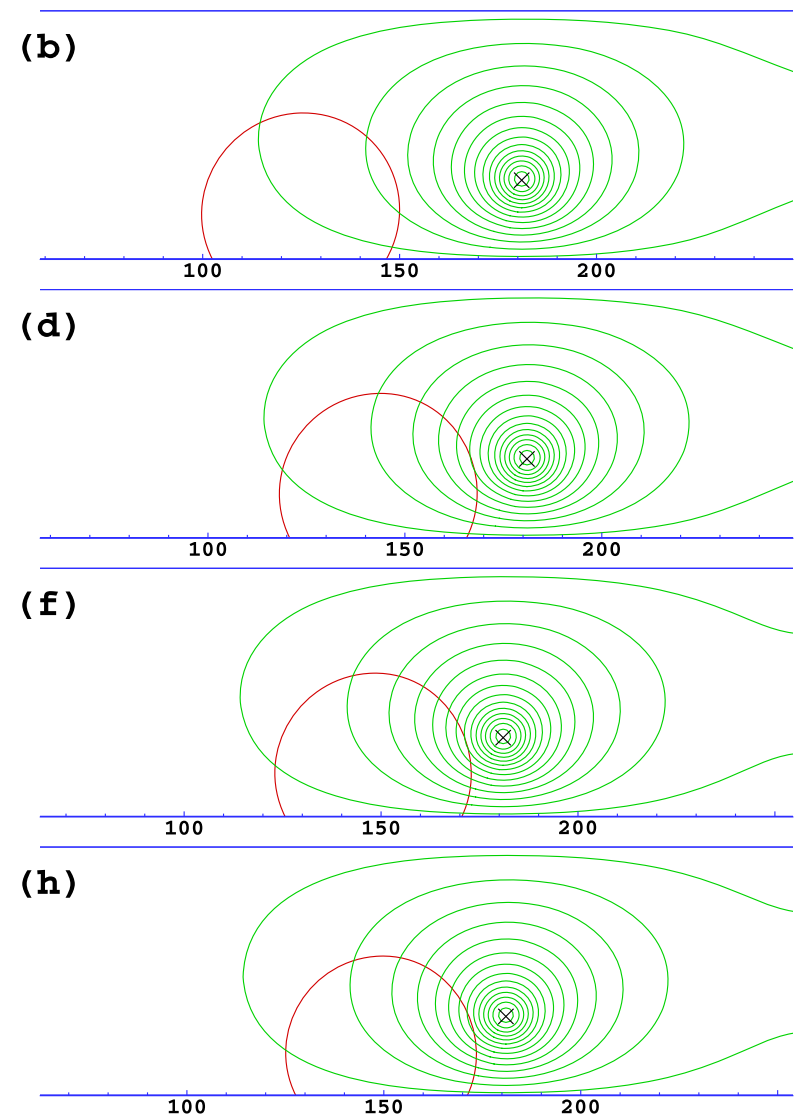

Fig. 13. (Color online.) The time evolution of the temperature field surrounding the moving droplet for $\theta^{e q}=120^{\circ}$ and $\lambda=1$ with the times taken as (a) $t^{*}=3.13$, (b) $t^{*}=9.69$, (c) $t^{*}=12.5$, (d) $t^{*}=14.38$, (e) $t^{*}=16.25$, (f) $t^{*}=18.75$, (g) $t^{*}=23.44$, and (h) $t^{*}=39.06$. At each time step, the zero contour of $\phi$ is indicated by the red solid line, and the temperature contours are plotted by the green solid lines with every 1.5 spacing and the initial value of 0.5 starting from the far field towards the center of the heating source, which is represented by an X-shaped cross.

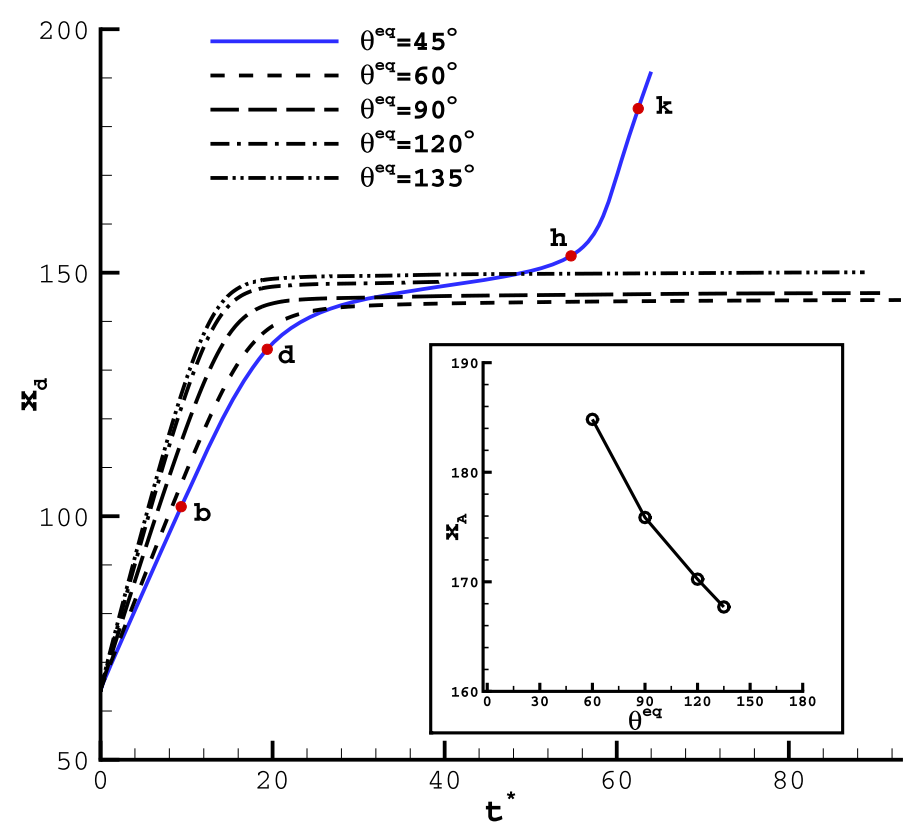

Fig. 14. (Color online.) The $x$-coordinate of droplet centroid as a function of dimensionless time for various contact angles, where the discrete points b, $d$, $h$ and $\mathrm{k}$ are explained in the text. The lower inset indicates the rest position of the advancing contact point as a function of the contact angle for $\theta^{\text {eq }} \geqslant 60^{\circ}$, where the droplet motion is completely blocked. 


\section{(a)}
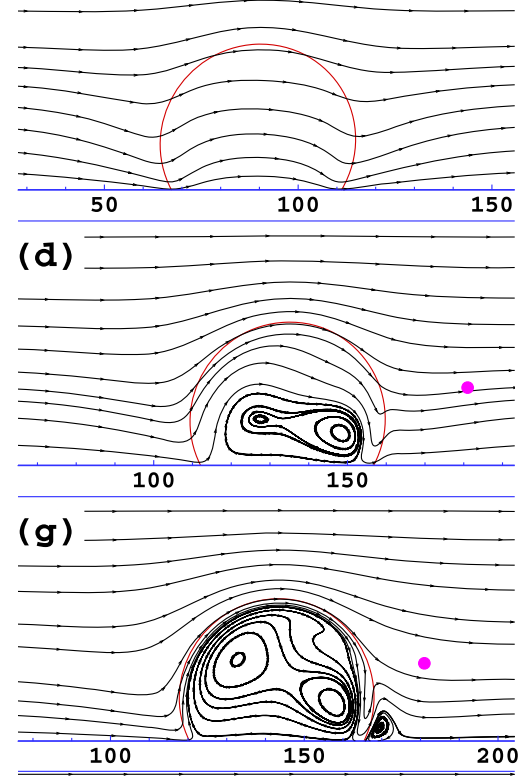

(j)

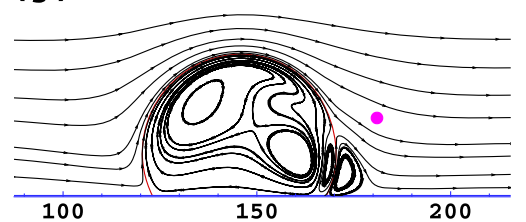

(b)
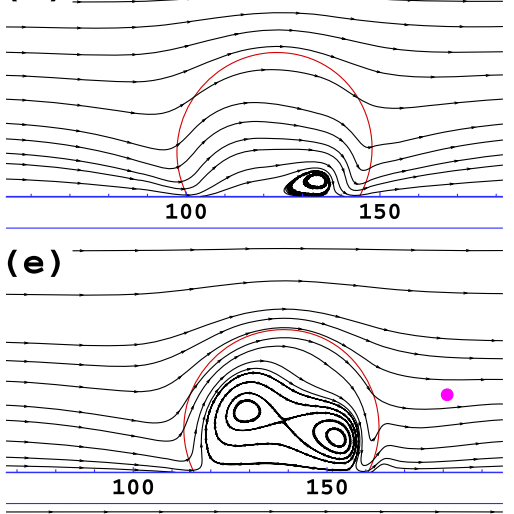

(h)

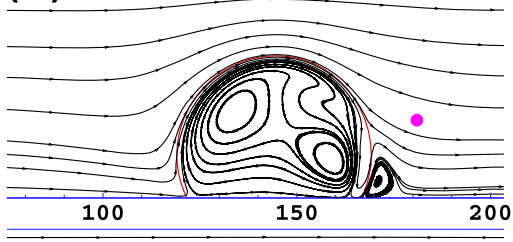

(k)

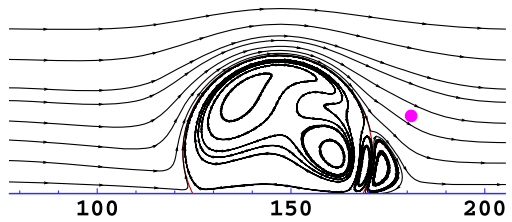

(c)

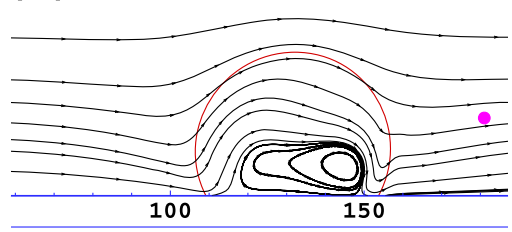

(f)

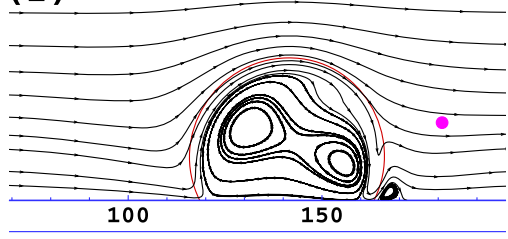

(i)

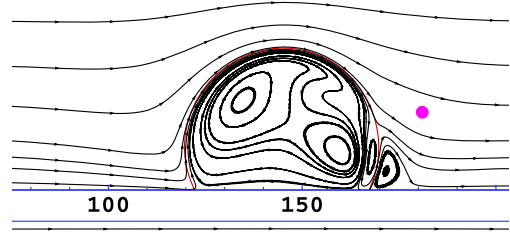

(1)

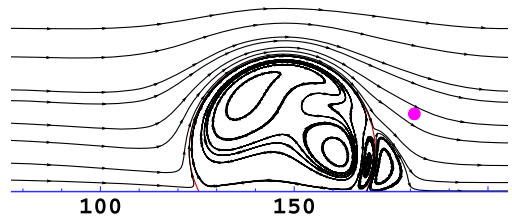

Fig. 15. (Color online.) The time evolution of droplet shape, position and streamlines surrounding the droplet for $\lambda=1 / 20$ with the times taken as: (a) $t^{*}=$ 3.13, (b) $t^{*}=7.81$, (c) $t^{*}=9.38$, (d) $t^{*}=10.0$, (e) $t^{*}=10.94$, (f) $t^{*}=11.88$, (g) $t^{*}=12.81$, (h) $t^{*}=13.75$, (i) $t^{*}=14.38$, (j) $t^{*}=15.63$, (k) $t^{*}=28.13$, and (l) $t^{*}=46.88$. The red solid lines are zero contours of the order parameter $\phi$, the black lines with arrows are the streamlines, and the pink point is the center of the heating source.

fluid-surface interactions. Therefore, we examine the effect of viscosity ratio for the fixed contact angle $\theta^{\text {eq }}=120^{\circ}$. Different viscosity ratios are achieved by adjusting the viscosity of dispersed phase while keeping the viscosity of continuous phase fixed. When the viscosity ratio is varied from $\frac{1}{20}$ to 10 , the droplet motion also undergoes two states. At low viscosity ratios, i.e., $\lambda \leqslant 1$, the droplet motion can be completely blocked; whereas at high viscosity ratios the droplet motion can only partially blocked, and droplet will eventually pass through the CHS. Although the droplet behavior is very similar for all $\lambda \leqslant 1$, the structures of internal vortices are significantly different, which can be seen from the comparison between $\lambda=\frac{1}{20}$ and $\lambda=1$. Note that the simulation result with $\lambda=1$ is not shown here because the growth and structure of all vortices exhibit similar characteristics to those given in Figs. 11 and 12, where two counter-rotating internal vortices completely fills the droplet, and the internal main vortex has much larger size. Fig. 15 gives the time evolution of droplet shape, position, and streamline patterns around the moving droplet for $\lambda=\frac{1}{20}$. At $t^{*}=7.81$, we can observe that a clockwise-rotating vortex starts to appear at the bottom wall inside the droplet, and it is closer to the front end of moving droplet compared to those in the cases with $\lambda=1$. As the droplet moves forward, the internal vortex becomes bigger, meanwhile it is elongated backwards due to large flow resistance, arising primarily from the thermocapillary convection at the front interface of droplet (see Fig. 15(c)). At $t^{*}=10.0$, another new vortex (called the $\mathrm{N}$ vortex hereafter) is formed on the left side inside the former internal vortex (called the F vortex hereafter), and it has a higher center in spatial position than the $\mathrm{F}$ vortex. As shown in Fig. 15(f), the N vortex significantly increases in size, and an external vortex arises at the wall surface near the front end of droplet interface. Subsequently, the droplet continues to advance towards the heating source, but the advancing velocity is relatively low. The external vortex and the $\mathrm{N}$ vortex both increase continuously in size, but the size of $\mathrm{F}$ vortex almost remains unchanged (see Fig. $15(\mathrm{~g})$ and $(\mathrm{h})$ ). In addition, it can be clearly seen that in the droplet, the streamlines adjacent to the CHS gradually become warped, indicating that the thermo-induced Marangoni convection is getting stronger. As the droplet approaches the steady state, an anticlockwise-rotating vortex appears between the external vortex and the F vortex (see Fig. 15(i)), as observed previously in Figs. 11 and 12. This new born vortex keeps growing slightly until $t^{*}=28.13$. Finally, the droplet motion creases completely and the system reaches the steady state (see Fig. 15(k) and (l)).

When the viscosity ratio is increased to 5, the induced thermocapillary force is not strong enough to stop the droplet movement, which can be clearly seen from Fig. 16 . At $t^{*}=12.81$, we can see a vortex formed at the bottom wall in the 

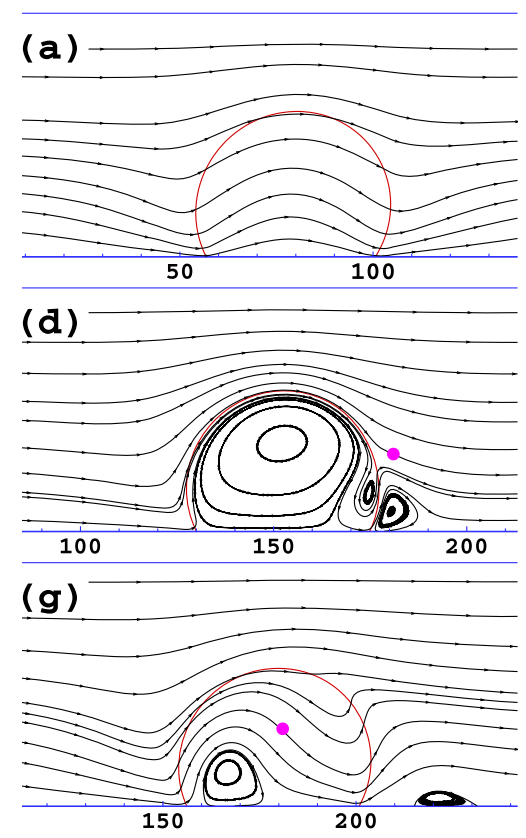
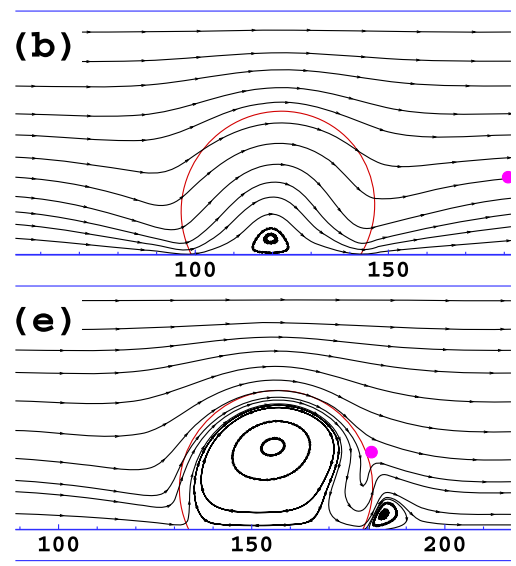

(h)

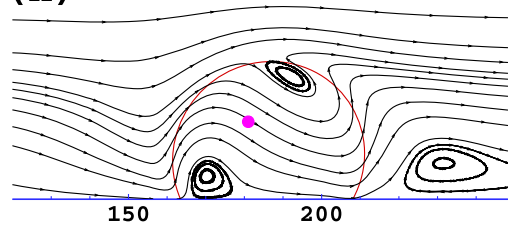

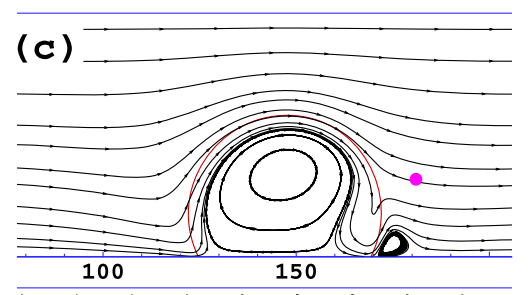
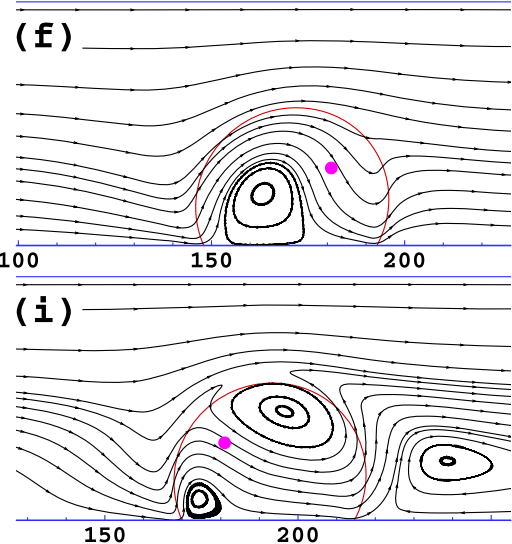

Fig. 16. (Color online.) The time evolution of droplet shape, position and streamlines surrounding the droplet for $\lambda=5$ with the times taken as: (a) $t^{*}=3.13$, (b) $t^{*}=12.81$, (c) $t^{*}=20.31$, (d) $t^{*}=23.44$, (e) $t^{*}=25.63$, (f) $t^{*}=28.13$, (g) $t^{*}=29.69$, (h) $t^{*}=31.25$, and (i) $t^{*}=32.81$. The red solid lines are zero contours of the order parameter $\phi$, the black lines with arrows are the streamlines, and the pink point is the center of the heating source.

middle of the contact line. Obviously, the vortex formation occurs much later compared with the case of $\lambda=1 / 20$, which is due to the fact that droplet moves slower at high viscosity ratios (see Fig. 17 below). This vortex, referred to as the main vortex, continuously increases in size, and an external vortex also starts to grow near the advancing contact point at $t^{*}=20.31$. The main and external vortices both keep growing until their sizes reach the maximum at $t^{*}=23.44$, when another internal vortex is found to arise between the two former vortices. However, the newborn internal vortex vanishes shortly, different from what we have seen in Fig. 10, where the droplet motion is also partially blocked. It should be noted that at $t^{*}=23.44$ the droplet front is just before the center of the heating source, beyond which the droplet motion will slow down. As the droplet front passes through the center of the heating source, all the vortices diminish (see Fig. 16(e) and (f)), and we can observe that the external vortex has disappeared at $t^{*}=28.13$. After that, the main vortex continues to shrink (see Fig. 16(g)-(i)). At $t^{*}=29.69$, a new wall vortex appears away from the advancing contact point around 15 lattices. Note that such a wall vortex is not observed in Fig. 10. This difference may be caused by the difference in contact angle. The wall vortex quickly increases (see Fig. 16(h) and (i)) and an internal vortex emerges near the top of the droplet (Fig. 16(h)), which is attributed to the Marangoni convection induced at the top interface of the droplet. The top vortex also grows quickly with its size much bigger than the main vortex at $t^{*}=32.81$.

To know the influence of viscosity ratio on the droplet motion, we plots the $x$-coordinate of droplet centroid as a function of dimensionless time for the viscosity ratios of $1 / 20,1 / 5,1,5$ and 10, which is shown in Fig. 17. For low viscosity ratios, i.e., $\lambda \leqslant 1$, the droplet first moves at a constant velocity, and then decelerates until it comes to rest. We interestingly observe that the droplet motion almost exhibits the same behavior for two lowest viscosity ratios. For high viscosity ratios, e.g., $\lambda \geqslant 5$, the droplet motion successively experiences the transition of CV, DC, AC, DC, ACV, DC, AC, DC, and CV. The former three regimes/processes, i.e., CV, DC and AC, are essentially the same as those observed in the case of $\theta^{e q}=45^{\circ}$ (see the blue line in Fig. 14), where the droplet is also blocked partially. We do observe an intermediate DC process (the open circle $\mathrm{f}$ in Fig. 17 with the corresponding snapshot given in Fig. 16(f)) when the droplet motion transits from AC to ACV; whereas for $\theta^{e q}=45^{\circ}$ the DC process is not clearly distinguished. The DC process results from the reduction in Marangoni force on the right side of the CHS, since the droplet front moves away from the region of high temperature gradient (see Fig. 16(f)). When the droplet interface on the left and right sides is approximately symmetric with respect to the CHS, the droplet motion will revert to an approximately constant velocity (see Fig. 16(g), which is the corresponding snapshot of open circle g in Fig. 17). As the droplet continues to advance, the interface symmetry is broken, and the interface on the left of CHS is closer to the CHS where the temperature gradient is higher, so the droplet once again decelerates. Once the whole droplet moves to the right of the CHS, the Marangoni force will contribute to accelerate its motion. As the droplet moves away from the CHS, the decreased Marangoni force cannot keep it moving at an increasing or even a constant velocity. Thus the droplet is slowed down due to the viscous stresses from the carrier fluid, and finally moves at constant velocity, as observed in the early stage. In addition, it can be noticed in Fig. 17 that, as the viscosity ratio decreases, the initial droplet velocity increases, but the droplet blocking becomes increasingly significant. This suggests that the Marangoni convection 


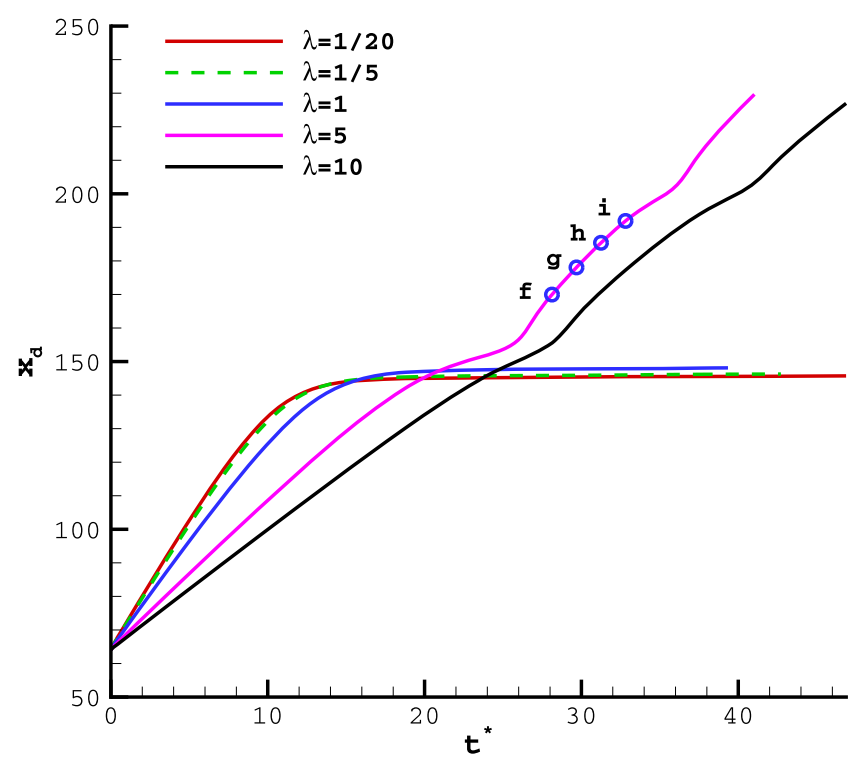

Fig. 17. (Color online.) The $x$-position of droplet centroid as a function of dimensionless time for various viscosity ratios. Note that the open circles on line of $\lambda=5$ correspond to the subfigures (f)-(i) in Fig. 16 .

is strengthened with decreasing viscosity ratio, which is consistent with the previous theoretical analysis and numerical results $[93,94]$.

\section{Conclusions}

A lattice Boltzmann phase-field model has been developed to simulate thermocapillary flows in a confined microfluidic geometry. Based on the phase-field theory, an interfacial force of potential form is proposed to model the interfacial tension forces and the Marangoni stresses because of the gradient of interfacial tension. As only the first-order derivatives are involved, the interfacial force of potential form can be easily combined with a cubic wetting boundary condition to account for the fluid-surface interactions, which can effectively suppress the spurious velocities near the solid walls. The hydrodynamic equations are solved through a multiple-relaxation-time approach, which can enhance the numerical stability for solving a binary fluid with large viscosity difference and produce correct equilibrium contact angles. The temperature field, which is coupled to the interfacial tension by the equation of state, is also solved in the LB framework, where thermal boundary conditions are imposed by a general halfway bounce-back scheme recently proposed by Zhang et al. [61].

The lattice Boltzmann phase-field model is validated and then used to numerically demonstrate that the laser heating can generate the thermocapillary forces to block the droplet motion when a liquid droplet is attached to a solid substrate in microchannel, subject to a simple shear flow. The position and intensity of the laser heating source are kept constant, and the thermal conductivity ratio of both fluids is fixed at $\chi=1$. When the contact angle $\theta^{\text {eq }}$ increases from $45^{\circ}$ to $135^{\circ}$, the droplet motion will transit from partial blocking to complete blocking, and the blocking becomes increasingly pronounced. In the case of partial blocking $\left(\theta^{e q}=45^{\circ}\right)$, the droplet motion successively experiences four processes: CV, DC, $A C$, and ACV. In the case of complete blocking $\left(\theta^{e q} \geqslant 60^{\circ}\right)$, the droplet firstly slides on the substrate at a constant velocity, and then slows down until it creases. When the droplet finally becomes motionless, the structure of vortices exhibits common characteristics: two counter-rotating vortices fill the droplet completely with the internal main vortex dominating in size, and an external vortex is situated at the corner of the droplet front and the bottom wall. The viscosity ratio $\lambda$ is also found to strongly influence the droplet dynamic behavior. For low viscosity ratios $(\lambda \leqslant 1)$, the droplet motion can be blocked completely. Although the droplet motion exhibits the similar behavior for all $\lambda \leqslant 1$, the structure of the internal vortices is more complicated for lower viscosity ratio. For high viscosity ratios $(\lambda \geqslant 5)$, the droplet motion is partially blocked and successively experiences the transition of CV, DC, AC, DC, ACV, DC, AC, DC, and CV, which is much more complex than what we observe in the case of $\theta^{e q}=45^{\circ}$. These transitions can be explained as a result of the competition between the Marangoni forces and viscous stresses, which are dynamically varying as the droplet moves forward.

\section{Acknowledgements}

This paper is based upon work supported by the LDRD Program (No. 20100025DR) of the Los Alamos National laboratory. 


\section{References}

[1] H. Song, D.L. Chen, R.F. Ismagilov, Reactions in droplets in microfluidic channels, Angew. Chem., Int. Ed. 45 (2006) 7336-7356.

[2] B.T.C. Lau, C.A. Baitz, X.P. Dong, C.L. Hansen, A complete microfluidic screening platform for rational protein crystallization, J. Am. Chem. Soc. 129 (3) (2007) 454-455.

[3] L. Li, R.F. Ismagilov, Protein crystallization using microfluidic technologies based on valves, droplets, and slipchip, Annu. Rev. Biophys. 39 (2010) $139-158$.

[4] P.S. Dittrich, A. Manz, Lab-on-a-chip: microfluidics in drug discovery, Nat. Rev. Drug Discov. 5 (2006) 210-218.

[5] Q. Xu, M. Hashimoto, T.T. Dang, T. Hoare, D.S. Kohane, G.M. Whitesides, R. Langer, D.G. Anderson, Preparation of monodisperse biodegradable polymer microparticles using a microfluidic flow-focusing device for controlled drug delivery, Small 5 (13) (2009) 1575-1581.

[6] C. Serra, N. Berton, M. Bouquey, L. Prat, G. Hadziioannou, A predictive approach of the influence of the operating parameters on the size of polymer particles synthesized in a simplified microfluidic system, Langmuir 23 (14) (2007) 7745-7750.

[7] N.J. Carroll, S.B. Rathod, E. Derbins, S. Mendez, D.A. Weitz, D.N. Petsev, Droplet-based microfluidics for emulsion and solvent evaporation synthesis of monodisperse mesoporous silica microspheres, Langmuir 24 (2008) 658-661.

[8] D.T. Chiu, R.M. Lorenz, Chemistry and biology in femtoliter and picoliter volume droplets, Acc. Chem. Res. 42 (5) (2009) 649-658.

[9] H. Zhang, G. Jenkins, Y. Zou, Z. Zhu, C.J. Yang, Massively parallel single-molecule and single-cell emulsion reverse transcription polymerase chain reaction using agarose droplet microfluidics, Anal. Chem. 84 (8) (2012) 3599-3606.

[10] C.-G. Yang, Z.-R. Xu, J.-H. Wang, Manipulation of droplets in microfluidic systems, Trends Anal. Chem. 29 (2) (2010) 141-157.

[11] A.R. Wheeler, Putting electrowetting to work, Science 322 (5901) (2008) 539-540.

[12] J. Gong, C.-J. Kim, All-electronic droplet generation on-chip with real-time feedback control for EWOD digital microfluidics, Lab Chip 8 (2008) $898-906$.

[13] J.R. Millman, K.H. Bhatt, B.G. Prevo, O.D. Velev, Anisotropic particle synthesis in dielectrophoretically controlled microdroplet reactors, Nat. Mater. 4 (2005) 98-102.

[14] K. Ahn, C. Kerbage, T.P. Hunt, R.M. Westervelt, D.R. Link, D.A. Weitz, Dielectrophoretic manipulation of drops for high-speed microfluidic sorting devices, Appl. Phys. Lett. 88 (2006) 024104.

[15] T. Thorsen, R.W. Roberts, F.H. Arnold, S.R. Quake, Dynamic pattern formation in a vesicle-generating microfluidic device, Phys. Rev. Lett. 86 (2001) 4163-4166.

[16] P. Garstecki, M.J. Fuerstman, H.A. Stone, G.M. Whitesides, Formation of droplets and bubbles in a microfluidic T-junction-scaling and mechanism of break-up, Lab Chip 6 (2006) 437-446.

[17] S.L. Anna, H.C. Mayer, Microscale tipstreaming in a microfluidic flow focusing device, Phys. Fluids 18 (2006) 121512.

[18] A.A. Darhuber, J.P. Valentino, J.M. Davis, S.M. Troian, S. Wagner, Microfluidic actuation by modulation of surface stresses, Appl. Phys. Lett. 82 (2003) 657.

[19] J.Z. Chen, S.M. Troian, A.A. Darhuber, S. Wagner, Effect of contact angle hysteresis on thermocapillary droplet actuation, J. Appl. Phys. 97 (2005) 014906.

[20] T. Franke, A.R. Abate, D.A. Weitz, A. Wixforth, Surface acoustic wave (SAW) directed droplet flow in microfluidics for PDMS devices, Lab Chip 9 (2009) $2625-2627$.

[21] M. Okochi, H. Tsuchiya, F. Kumazawa, M. Shikida, H. Honda, Droplet-based gene expression analysis using a device with magnetic force-based-droplethandling system, J. Biosci. Bioeng. 109 (2) (2010) 193-197.

[22] Y. Zhang, S. Park, K. Liu, J. Tsuan, S. Yang, T.-H. Wang, A surface topography assisted droplet manipulation platform for biomarker detection and pathogen identification, Lab Chip 11 (2011) 398-406.

[23] R. Di Leonardo, G. Ruocco, J. Leach, M.J. Padgett, A.J. Wright, J.M. Girkin, D.R. Burnham, D. McGloin, Parametric resonance of optically trapped aerosols, Phys. Rev. Lett. 99 (1) (2007) 010601.

[24] D. McGloin, D.R. Burnham, M.D. Summers, D. Rudd, N. Dewar, S. Anand, Optical manipulation of airborne particles: techniques and applications, Faraday Discuss. 137 (2008) 335-350.

[25] C. Baroud, M.R. de Saint Vincent, J.-P. Delville, An optical toolbox for total control of droplet microfluidics, Lab Chip 7 (2007) $1029-1033$.

[26] L.E. Scriven, C.V. Sternling, The Marangoni effects, Nature 187 (1960) 186-188.

[27] C. Baroud, J.-P. Delville, F. Gallaire, R. Wunenburger, Thermocapillary valve for droplet production and sorting, Phys. Rev. E 75 (2007) 046302.

[28] M. Robert de Saint Vincent, J.-P. Delville, Thermocapillary migration in small-scale temperature gradients: Application to optofluidic drop dispensing, Phys. Rev. E 85 (2012) 026310.

[29] R.S. Subramanian, R. Balasubramanian, The Motion of Bubbles and Drops in Reduced Gravity, Cambridge University Press, Cambridge, 2001.

[30] K. Kotz, K. Noble, G. Faris, Optical microfluidics, Appl. Phys. Lett. 85 (2004) 2658.

[31] M. Robert de Saint Vincent, R. Wunenburger, J.-P. Delville, Laser switching and sorting for high speed digital microfluidics, Appl. Phys. Lett. 92 (2008) 154105.

[32] C.M. Pooley, H. Kusumaatmaja, J.M. Yeomans, Contact line dynamics in binary lattice Boltzmann simulations, Phys. Rev. E 78 (5) (2008) 056709.

[33] D. Juric, G. Tryggvason, A front-tracking method for dendritic solidification, J. Comput. Phys. 123 (1) (1996) 127-148.

[34] W. Shyy, R.W. Smith, H.S. Udaykumar, M.M. Rao, Computational Fluid Dynamics with Moving Boundaries, Taylor \& Francis, Washington, DC, 1996.

[35] H.-Y. Chen, D. Jasnow, J. Viñals, Interface and contact line motion in a two phase fluid under shear flow, Phys. Rev. Lett. 85 (2000) $1686-1689$.

[36] S. Chen, G.D. Doolen, Lattice Boltzmann method for fluid flows, Annu. Rev. Fluid Mech. 30 (1) (1998) 329-364.

[37] A.K. Gunstensen, D.H. Rothman, S. Zaleski, G. Zanetti, Lattice Boltzmann model of immiscible fluids, Phys. Rev. A 43 (8) (1991) $4320-4327$.

[38] D.H. Rothman, J.M. Keller, Immiscible cellular-automaton fluids, J. Stat. Phys. 52 (1988) 1119-1127.

[39] D. Grunau, S. Chen, K. Chen, A lattice Boltzmann model for multi-phase fluid flows, Phys. Fluids A 5 (1993) 2557-2562.

[40] T. Reis, T.N. Phillips, Lattice Boltzmann model for simulating immiscible two-phase flows, J. Phys. A, Math. Theor. 40 (14) (2007) $4033-4053$.

[41] H. Liu, A.J. Valocchi, Q. Kang, Three-dimensional lattice Boltzmann model for immiscible two-phase flow simulations, Phys. Rev. E 85 (2012) 046309.

[42] X. Shan, H. Chen, Lattice Boltzmann model for simulating flows with multiple phases and components, Phys. Rev. E 47 (3) (1993) $1815-1819$.

[43] X. Shan, H. Chen, Simulation of nonideal gases and liquid-gas phase transitions by the lattice Boltzmann equation, Phys. Rev. E 49 (1994) $2941-2948$.

[44] M. Sbragaglia, R. Benzi, L. Biferale, S. Succi, K. Sugiyama, F. Toschi, Generalized lattice Boltzmann method with multirange pseudopotential, Phys. Rev. E 75 (2007) 026702.

[45] M.R. Swift, W.R. Osborn, J.M. Yeomans, Lattice Boltzmann simulation of nonideal fluids, Phys. Rev. Lett. 75 (5) (1995) 830-833.

[46] M.R. Swift, E. Orlandini, W.R. Osborn, J.M. Yeomans, Lattice Boltzmann simulations of liquid-gas and binary fluid systems, Phys. Rev. E 54 (5) (1996) $5041-5052$.

[47] T. Lee, C.-L. Lin, A stable discretization of the lattice Boltzmann equation for simulation of incompressible two-phase flows at high density ratio, J. Comput. Phys. 206 (1) (2005) 16-47.

[48] H. Zheng, C. Shu, Y. Chew, A lattice Boltzmann model for multiphase flows with large density ratio, J. Comput. Phys. 218 (1) (2006) $353-371$.

[49] T. Lee, L. Liu, Lattice Boltzmann simulations of micron-scale drop impact on dry surfaces, J. Comput. Phys. 229 (20) (2010) 8045-8063.

[50] X. He, S. Chen, R. Zhang, A lattice Boltzmann scheme for incompressible multiphase flow and its application in simulation of Rayleigh-Taylor instability, J. Comput. Phys. 152 (2) (1999) 642-663. 
[51] R.R. Nourgaliev, T.N. Dinh, T.G. Theofanous, D. Joseph, The lattice Boltzmann equation method: theoretical interpretation, numerics and implications, Int. J. Multiph. Flow 29 (1) (2003) 117-169.

[52] C.K. Aidun, J.R. Clausen, Lattice-Boltzmann method for complex flows, Annu. Rev. Fluid Mech. 42 (1) (2010) 439-472.

[53] H. Liu, Y. Zhang, A.J. Valocchi, Modeling and simulation of thermocapillary flows using lattice Boltzmann method, J. Comput. Phys. 231 (12) (2012) 4433-4453.

[54] H. Liu, A.J. Valocchi, Y. Zhang, Q. Kang, A phase-field-based lattice-Boltzmann finite-difference model for simulating thermocapillary flows, Phys. Rev. E 87 (2013) 013010.

[55] M. Latva-Kokko, D.H. Rothman, Diffusion properties of gradient-based lattice Boltzmann models of immiscible fluids, Phys. Rev. E 71 (2005) 056702.

[56] D. Jacqmin, Calculation of two-phase Navier-Stokes flows using phase-field modeling, J. Comput. Phys. 155 (1999) 96-127.

[57] D. Jamet, D. Torres, J.U. Brackbill, On the theory and computation of surface tension: The elimination of parasitic currents through energy conservation in the second-gradient method, J. Comput. Phys. 182 (1) (2002) 262-276.

[58] T. Lee, P.F. Fischer, Eliminating parasitic currents in the lattice Boltzmann equation method for nonideal gases, Phys. Rev. E 74 (4) (2006) 046709.

[59] Z. Yu, L.-S. Fan, Multirelaxation-time interaction-potential-based lattice Boltzmann model for two-phase flow, Phys. Rev. E 82 (2010) 046708.

[60] M.L. Porter, E.T. Coon, Q. Kang, J.D. Moulton, J.W. Carey, Multicomponent interparticle-potential lattice Boltzmann model for fluids with large viscosity ratios, Phys. Rev. E 86 (2012) 036701

[61] T. Zhang, B. Shi, Z. Guo, Z. Chai, J. Lu, General bounce-back scheme for concentration boundary condition in the lattice-Boltzmann method, Phys. Rev. E 85 (2012) 016701.

[62] D. Jacqmin, Contact-line dynamics of a diffuse fluid interface, J. Fluid Mech. 402 (2000) 57-88.

[63] V.V. Khatavkar, P.D. Anderson, H.E.H. Meijer, Capillary spreading of a droplet in the partially wetting regime using a diffuse-interface model, J. Fluid Mech. 572 (2007) 367-387.

[64] H. Wiklunda, S. Lindströmb, T. Uesaka, Boundary condition considerations in lattice Boltzmann formulations of wetting binary fluids, Comput. Phys. Commun. 182 (2011) 2192-2200.

[65] W. Villanueva, G. Amberg, Some generic capillary-driven flows, Int. J. Multiph. Flow 32 (2006) 1072-1086.

[66] V.V. Khatavkar, P.D. Anderson, P.C. Duineveld, H.E.H. Meijer, Diffuse-interface modelling of droplet impact, J. Fluid Mech. 581 (2007) $97-127$.

[67] H. Ding, P.D.M. Spelt, Wetting condition in diffuse interface simulations of contact line motion, Phys. Rev. E 75 (2007) 046708.

[68] C. Liu, J. Shen, A phase field model for the mixture of two incompressible fluids and its approximation by a Fourier-spectral method, Physica D 179 (3-4) (2003) 211-228.

[69] C. Ma, D. Bothe, Direct numerical simulation of thermocapillary flow based on the Volume of Fluid method, Int. J. Multiph. Flow 37 (9) (2011) $1045-1058$.

[70] P. Lallemand, L.-S. Luo, Theory of the lattice Boltzmann method: Acoustic and thermal properties in two and three dimensions, Phys. Rev. E 68 (2003) 036706.

[71] C. Pan, L.-S. Luo, C.T. Miller, An evaluation of lattice Boltzmann schemes for porous medium flow simulation, Comput. Fluids 35 (2006) 898-909.

[72] D. d'Humières, I. Ginzburg, M. Krafczyk, P. Lallemand, L.-S. Luo, Multiple-relaxation-time lattice Boltzmann models in three dimensions, Philos. Trans. R. Soc. A 360 (2002) 437-451.

[73] P. Lallemand, L.-S. Luo, Theory of the lattice Boltzmann method: Dispersion, dissipation, isotropy, Galilean invariance, and stability, Phys. Rev. E 61 (6) (2000) 6546-6562.

[74] M.E. McCracken, J. Abraham, Multiple-relaxation-time lattice-Boltzmann model for multiphase flow, Phys. Rev. E 71 (2005) 036701.

[75] L.-S. Luo, W. Liao, X. Chen, Y. Peng, W. Zhang, Numerics of the lattice Boltzmann method: Effects of collision models on the lattice Boltzmann simulations, Phys. Rev. E 83 (2011) 056710.

[76] Z. Guo, T.S. Zhao, A lattice Boltzmann model for convection heat transfer in porous media, Numer. Heat Transf., B Fundam. 47 (2005) $157-177$.

[77] X. Shan, Simulation of Rayleigh-Bénard convection using a lattice Boltzmann method, Phys. Rev. E 55 (1997) 2780-2788.

[78] Y. Peng, C. Shu, Y. Chew, Simplified thermal lattice Boltzmann model for incompressible thermal flows, Phys. Rev. E 68 (2003) 026701.

[79] A.J.C. Ladd, Numerical simulations of particulate suspensions via a discretized Boltzmann equation. (Part I \& II), J. Fluid Mech. 271 (1994) $285-339$.

[80] A.J. Briant, P. Papatzacos, J.M. Yeomans, Lattice Boltzmann simulations of contact line motion in a liquid-gas system, Philos. Trans. R. Soc. A 360 (2002) 485-495.

[81] B. Pendse, A. Esmaeeli, An analytical solution for thermocapillary-driven convection of superimposed fluids at zero Reynolds and Marangoni numbers, Int. J. Therm. Sci. 49 (7) (2010) 1147-1155.

[82] R. Du, B. Shi, X. Chen, Multi-relaxation-time lattice Boltzmann model for incompressible flow, Phys. Lett. A 359 (2006) 564-572.

[83] P. Lallemand, L.-S. Luo, Y. Peng, A lattice Boltzmann front-tracking method for interface dynamics with surface tension in two dimensions, J. Comput. Phys. 226 (2007) 1367-1384.

[84] A. Kuzmin, A.A. Mohamad, S. Succi, Multi-relaxation time lattice Boltzmann model for multiphase flows, Int. J. Mod. Phys. C 6 (2008) 875-902.

[85] J.-J. Huang, H. Huang, C. Shu, Y.T. Chew, S.-L. Wang, Hybrid multiple-relaxation-time lattice-Boltzmann finite-difference method for axisymmetric multiphase flows, J. Phys. A, Math. Theor. 46 (2013) 055501.

[86] Q. Li, K.H. Luo, X.J. Li, Lattice Boltzmann modeling of multiphase flows at large density ratio with an improved pseudopotential model, Phys. Rev. E 87 (2013) 053301.

[87] A. Fakhari, T. Lee, Multiple-relaxation-time lattice Boltzmann method for immiscible fluids at high Reynolds numbers, Phys. Rev. E 87 (2013) 023304.

[88] I. Ginzburg, Equilibrium-type and link-type lattice Boltzmann models for generic advection and anisotropic-dispersion equation, Adv. Water Resour. 28 (2005) 1171-1195.

[89] E. Lajeunesse, G.M. Homsy, Thermocapillary migration of long bubbles in polygonal tubes. II. Experiments, Phys. Fluids 15 (2003) 308.

[90] H.-B. Nguyen, J.-C. Chen, A numerical study of thermocapillary migration of a small liquid droplet on a horizontal solid surface, Phys. Fluids 22 (2010) 062102.

[91] R. van der Sman, S. van der Graaf, Emulsion droplet deformation and breakup with lattice Boltzmann model, Comput. Phys. Commun. 178 (2008) 492-504.

[92] H. Liu, Y. Zhang, Phase-field modeling droplet dynamics with soluble surfactants, J. Comput. Phys. 229 (2010) 9166-9187.

[93] N. Young, J. Goldstein, M. Block, The motion of bubbles in a vertical temperature gradient, J. Fluid Mech. 6 (1959) 350-356.

[94] J.-F. Zhao, Z.-D. Li, H.-X. Li, J. Li, Thermocapillary migration of deformable bubbles at moderate to large Marangoni number in microgravity, Microgravity Sci. Technol. 22 (2010) 295-303. 